\title{
Cytotoxic CD4+ T lymphocytes may induce endothelial cell apoptosis in systemic sclerosis
}

\author{
Takashi Maehara, ${ }^{1,2}$ Naoki Kaneko, ${ }^{1,2}$ Cory A. Perugino, ${ }^{1,3}$ Hamid Mattoo, ${ }^{1,4}$ Jesper Kers, ${ }^{1,5,6,7}$ Hugues Allard-Chamard, ${ }^{1,8}$ \\ Vinay S. Mahajan, ${ }^{1,9}$ Hang Liu, ${ }^{1,0}$ Samuel J.H. Murphy, ${ }^{1}$ Musie Ghebremichael, ${ }^{1}$ David Fox, ${ }^{11}$ Aimee S. Payne, ${ }^{12}$ Robert Lafyatis, ${ }^{13}$ \\ John H. Stone, ${ }^{3}$ Dinesh Khanna, ${ }^{11}$ and Shiv Pillai' \\ 1'Ragon Institute, Massachusetts General Hospital, Massachusetts Institute of Technology and Harvard University, Boston, Massachusetts, USA. ${ }^{2}$ Section of Oral and Maxillofacial Oncology, Division of \\ Maxillofacial Diagnostic and Surgical Sciences, Faculty of Dental Science, Kyushu University, Fukuoka, Japan. ${ }^{3}$ Division of Rheumatology, Allergy and Immunology, Massachusetts General Hospital, Boston, \\ Massachusetts, USA. ${ }^{4}$ Immunology and Inflammation Therapeutic Area, Sanofi, Cambridge Massachusetts, USA. ${ }^{5}$ Amsterdam Infection \& Immunity Institute (Al\&II) and ${ }^{6}$ Amsterdam Cardiovascular \\ Sciences (ACS), Amsterdam University Medical Centers, and ${ }^{7}$ Van 't Hoff Institute for Molecular Sciences (HIMS), University of Amsterdam, Amsterdam, Netherlands. ${ }^{8}$ Division of Rheumatology, Faculté de \\ médecine et des sciences de la santé, Université de Sherbrooke et Centre de Recherche Clinique Étienne-Le Bel, Sherbrooke, Québec, Canada. ${ }^{9}$ Department of Pathology, Brigham and Women's Hospital, \\ Boston, Massachusetts, USA. ${ }^{10}$ Department of Rheumatology and Immunology, First Affiliated Hospital of China, Shenyang, China. "Division of Rheumatology, Medical School, University of Michigan, \\ Ann Arbor, Michigan, USA. ${ }^{12}$ Department of Dermatology, Perelman School of Medicine, University of Pennsylvania, Philadelphia, Pennsylvania, USA. ${ }^{13}$ Division of Rheumatology and Clinical Immunology, \\ Department of Medicine, School of Medicine, University of Pittsburgh, Pittsburgh, Pennsylvania, USA.
}

Systemic sclerosis (SSc) is an autoimmune fibrotic disease whose pathogenesis is poorly understood and lacks effective therapies. We undertook quantitative analyses of $\mathrm{T}$ cell infiltrates in the skin of 35 untreated patients with early diffuse SSc and here show that $\mathrm{CD} 4^{+}$cytotoxic $\mathrm{T}$ cells and $\mathrm{CD} 8^{+} \mathrm{T}$ cells contribute prominently to these infiltrates. We also observed an accumulation of apoptotic cells in SSc tissues, suggesting that recurring cell death may contribute to tissue damage and remodeling in this fibrotic disease. HLA-DR-expressing endothelial cells were frequent targets of apoptosis in SSc, consistent with the prominent vasculopathy seen in patients with this disease. A circulating effector population of cytotoxic CD4 ${ }^{+} \mathrm{T}$ cells, which exhibited signatures of enhanced metabolic activity, was clonally expanded in patients with systemic sclerosis. These data suggest that cytotoxic T cells may induce the apoptotic death of endothelial and other cells in systemic sclerosis. Cell loss driven by immune cells may be followed by overly exuberant tissue repair processes that lead to fibrosis and tissue dysfunction.

\section{Introduction}

Systemic sclerosis (SSc) is a systemic autoimmune inflammatory disorder of unknown etiology characterized by prominent fibrosis that may affect many organs including the skin, lungs, kidneys, and gastrointestinal tract (1). Another important feature of this disease is vasculopathy that leads to vasospasm, pulmonary arterial remodeling, and microvascular obliteration (1-4). SSc is progressive, highly morbid, and in its most severe form carries a mortality rate comparable to the most fatal metastatic cancers (5). Current treatment paradigms slow fibrosis but do not halt or reverse it. True disease regression has only consistently been observed following autologous stem cell transplantation (6-9). The therapeutic efficacy of stem cell transplantation supports the notion that SSc is driven by an aberrant immune response and that the disease process can be reversed. Our imprecise understanding of the immune mechanisms that drive SSc likely

Authorship note: TM, NK, and CAP contributed equally to this work.

Conflict of interest: CAP, DK, and JHS are consultants to BMS. SP is on the Scientific Advisory Board (SAB) of Abpro Inc. ASP is cofounder, cochair of the SAB of Cabaletta Bio. Copyright: () 2020, American Society for Clinical Investigation.

Submitted: July 11, 2019; Accepted: January 23, 2020; Published: April 6, 2020.

Reference information: / Clin Invest. 2020;130(5):2451-2464.

https://doi.org/10.1172/JCl131700. contributes to the difficulty in identifying effective therapeutics in this disease.

$\mathrm{T}$ cells are present in SSc tissues and have been postulated to drive both the endothelial dysfunction and the fibrotic pathology of this disease, presumably via the secretion of cytokines that eventually contribute to the activation of macrophages, fibroblasts, and myofibroblasts (10). The latter 2 cell types, fibroblasts and myofibroblasts, secrete type I collagens and matrix metalloproteinases such as MMP-9 and MMP-12, and the process of dysregulated wound healing could contribute to the fibrosis seen in this disease. Whether fibrosis is a consequence of parenchymal cell death and tissue remodeling or results primarily from the cytokines within an inflammatory milieu is unclear. These mechanisms are not mutually exclusive, however, and could even be sequential.

In SSc, Th 2 cells, Th17 cells, Th1 cells, Th22 cells, Treg cells, T follicular helper (Tfh) cells, and $\mathrm{CD}^{+} \mathrm{T}$ cells have all been implicated in the pathogenic process (11-21). However, no previous studies have used quantitative approaches to enumerate $\mathrm{T}$ cell subsets within SSc lesions. There remains a paucity of information regarding the phenotype of infiltrating $\mathrm{T}$ cells and the precise role of $\mathrm{T}$ cells in the pathogenesis of this disease remains to be established. Although B cells have also been associated with the pathogenesis of SSc and beneficial effects of B cell-depleting therapy have been reported, the relative contributions of $B$ and 
T lymphocytes have not been systematically interrogated at the tissue level (22-24).

We chose to interrogate cytotoxic $\mathrm{CD} 4^{+} \mathrm{T}$ lymphocytes $\left(\mathrm{CD} 4^{+}\right.$ CTLs) and other CD4 $4^{+} \mathrm{T}$ cell subsets in SSc tissues based on our previous blood and tissue studies on IgG4-related disease (IgG4$\mathrm{RD})$. IgG4-RD is also a chronic, immune-mediated fibrotic disorder characterized by inflammatory tumor-like masses with a predilection for the lacrimal glands, salivary glands, pancreas, bile ducts, and retroperitoneal tissues (25). We have identified CD $4^{+}$ CTLs as the dominant effector $\mathrm{CD} 4^{+} \mathrm{T}$ cell subset infiltrating tissues affected by IgG4-RD along with large clonal expansions in the blood (26-28). These cells decline in conjunction with treatment-induced remission $(26,28) . \mathrm{CD}^{+} \mathrm{CTL}$ s have the potential to drive fibrosis through the elaboration of profibrotic cytokines and/ or by the induction of apoptosis of target cells (26).

CD4 ${ }^{+}$CTLs have been extensively studied in the setting of chronic viral infections such as CMV and HIV but there remain many gaps in our knowledge regarding these cells and their potential role in immune-mediated fibrosis (29-31). In addition to the in-depth investigation of CD $4^{+}$CTLs in IgG4-RD, these cells have also been associated with idiopathic pulmonary fibrosis and CD $4^{+}$ $\mathrm{T}$ cells with a cytotoxic phenotype have recently been reported in the context of rheumatoid arthritis $(32,33)$. Studies in healthy volunteers demonstrated heterogeneity of CD4 $4^{+}$CTLs among effector memory $\mathrm{T}$ cells that had regained CD45RA expression (Temra), and an IL-7 receptor-expressing subset was defined as the precursor population to effector $\mathrm{CD}^{+}$CTLs $(34,35)$. Functional subsets of $\mathrm{CD} 4^{+}$CTLs have, however, not been identified in the context of inflammation and human fibrotic disease. Moreover, although we had previously described CD $4^{+}$CTL expansions within the blood of a relatively small number of SSc patients, the relevance of these cells to the pathogenesis of SSc has not yet been investigated rigorously, and the broader relevance of CD $4^{+}$CTLs to diseases other than IgG4-RD requires exploration (26).

In our studies described here, we show that both $\mathrm{CD} 4^{+}$and $\mathrm{CD}^{+} \mathrm{T}$ cells accumulate in skin biopsies of untreated patients with early diffuse SSc and outnumber infiltrating B cells. In most of these patients $\mathrm{CD} 4^{+}$CTLs represent the dominant $\mathrm{CD} 4^{+} \mathrm{T}$ cell subset infiltrating the skin, whereas Th1, Th2, Tfh, and Treg cells are relatively sparse in SSc tissues. Examination of selected patients with lower numbers of infiltrating CD $4^{+}$CTLs revealed some increase in Th17 cells, but the dominant $\mathrm{T}$ cells in the overwhelming majority of SSc patients are cytotoxic T cells - including $\mathrm{CD}^{+}$CTLs and effector CD8 ${ }^{+} \mathrm{T}$ cells. We catalog the prominent apoptosis of endothelial cells, the accumulation of CD4 $4^{+}$CTLs in the vicinity of the endothelium, and the upregulation of HLA class II by endothelial cells in SSc skin biopsies, raising the possibility that $\mathrm{CD} 4^{+} \mathrm{CTL}$ may drive tissue fibrosis by contributing to the apoptotic death of specific cell types in an antigen-specific manner. In addition, $\mathrm{CD} 4^{+} \mathrm{CTLs}$ elaborate proinflammatory and profibrotic cytokines that may contribute to an overexuberant, cytokine-driven tissue remodeling process inducing the secretion of excessive amounts of collagen, thereby compromising tissue and organ function. We further categorize an effector subset of CD4 ${ }^{+}$ CTLs from the blood of SSc patients, denoted by the loss of surface CD28 and gain of CD57. This population of cells shows marked clonal expansion and a highly cytotoxic profile at both the protein and transcriptional levels. We also demonstrate that the accumulation of $\mathrm{CD}^{+}$CTLs in the skin of SSc patients can be attenuated by treatment with CTLA4-Ig, suggesting that ongoing $\mathrm{T}$ cell activation contributes to the pathologic alterations that are observed in this disease.

\section{Results}

$C D 4^{+} C T L$ s and $C D 8^{+} T$ cells are prominent in SSctissues. In our analyses of the adaptive immune cells infiltrating tissues affected by SSc, we simultaneously quantified the relative contribution of $\mathrm{CD}^{+} \mathrm{T}$ cells and $\mathrm{CD} 19^{+} \mathrm{B}$ cells in the skin of 35 patients with early diffuse SSc before the initiation of any immunosuppressive treatment. Although prominent numbers of both B and T cells were observed, the absolute number of infiltrating $\mathrm{T}$ cells was greater than those of infiltrating B cells (Figure 1, A and B). Although randomized double-blind clinical trials examining the utility of $\mathrm{B}$ cell depletion therapy in SSc have not been performed, some studies have reported clinical improvement with rituximab, especially in the context of early diffuse SSc $(22,23)$. Additionally, activated B cells have been observed in the circulation of SSc patients $(24,36)$. Although there may be a contribution of infiltrating B cells to pathology in $\mathrm{SSc}$, we focused on the T cells in the infiltrate across this group of patients given their relative preponderance in the tissue.

We first examined the relative contribution of $\mathrm{CD} 8^{+}$and $\mathrm{CD} 4^{+}$ $\mathrm{T}$ cells to the overall $\mathrm{CD}^{+} \mathrm{T}$ cell infiltrate. We found that $\mathrm{CD} 8^{+} \mathrm{T}$ cells and $\mathrm{CD}^{+} \mathrm{T}$ cells were both present in high numbers in SSc tissues, with some variation in the relative abundance of each of these $\mathrm{T}$ cell lineages across patients (Figure 1, C and D). Given the varied literature regarding $\mathrm{CD} 4^{+} \mathrm{T}$ cell subsets in the context of SSc, we directly quantified $\mathrm{CD} 4^{+} \mathrm{T}$ cell subsets in the skin across this cohort of 35 untreated patients. We initially defined CD4 ${ }^{+}$ CTLs by the coexpression of CD4 and granzyme A (GZMA) and quantified their relative contribution to the total $\mathrm{CD} 4^{+} \mathrm{T}$ cell infiltrate. Consistent with our previous report of expanded CD $4^{+}$CTLs in the blood of SSc patients (26), we observed a prominent accumulation of CD4 ${ }^{+}$CTLs within the affected skin of SSc patients (Figure 2, A-C). To validate this finding, we additionally costained tissues for CD4 and SLAMF7, a surface marker we have previously described in CD4 ${ }^{+}$CTLs (26). Consistently, we observed a marked expansion of SLAMF7-expressing CD $4^{+} \mathrm{T}$ cells in SSc skin samples along with the coexpression of SLAMF7 and GZMA (Supplemental Figure 1, A-C; supplemental material available online with this article; https://doi.org/10.1172/JCI131700DS1). Because multiple reports have associated Th2 cells with the pathogenesis of SSc, we first focused on the relative contribution of Th2 cells compared with $\mathrm{CD} 4^{+}$CTLs and observed a striking preponderance of $\mathrm{CD} 4^{+}$ CTLs in contrast to the small proportions and absolute numbers of Th2 cells (Figure 2, A-C and refs. 11-14). Utilizing skin samples from patients with bullous pemphigoid, an established Th2-mediated autoimmune skin disease, we compared the absolute numbers of CD $4^{+}$CTLs and Th2 cells across our experimental groups (37). Consistent with previous reports, Th2 cell numbers were greatly increased in bullous pemphigoid tissues and outnumbered those in SSc and healthy control skin (Figure 2C and Supplemental Figure 2). Despite the 5- to 10 -fold greater number of $\mathrm{CD}^{+} \mathrm{T}$ cells in skin biopsies of bullous pemphigoid patients compared with SSc biopsies, we found the absolute numbers of tissue-infiltrating 


\section{A CD3 CD19 DAP}
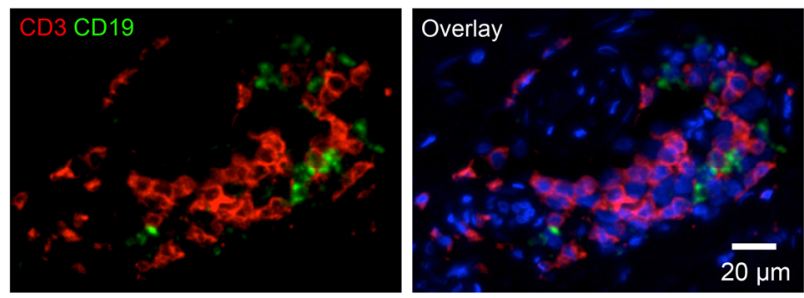

SSc

B

Tissue quantification

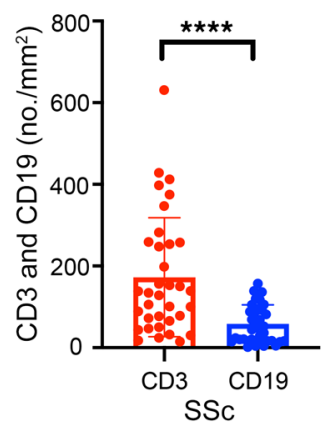

SSc

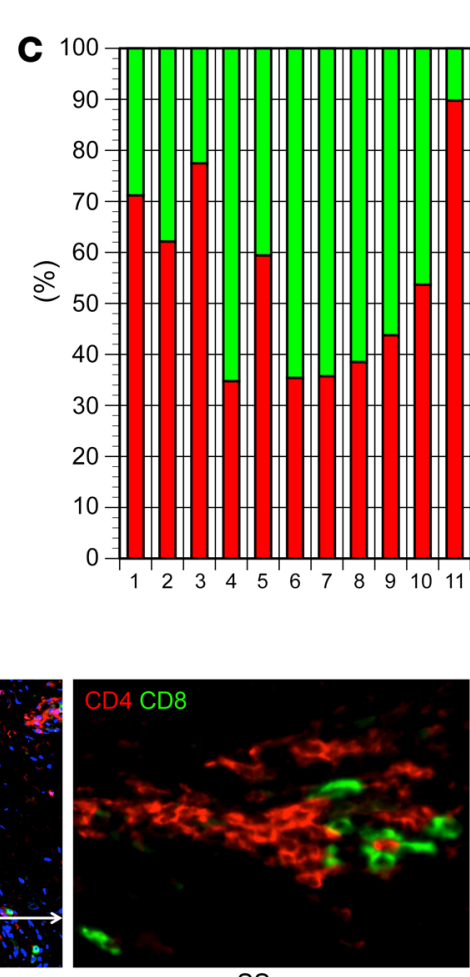

$\mathrm{SSc}$
Tissue quantification

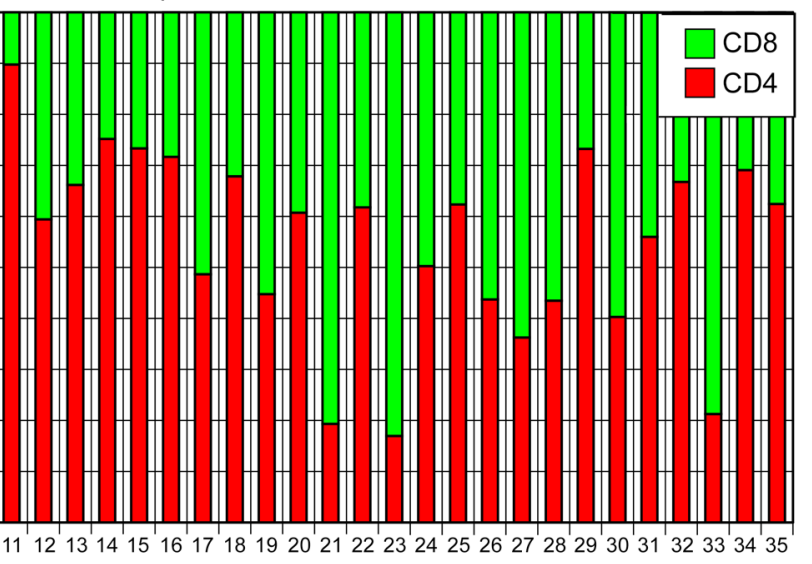

SSc

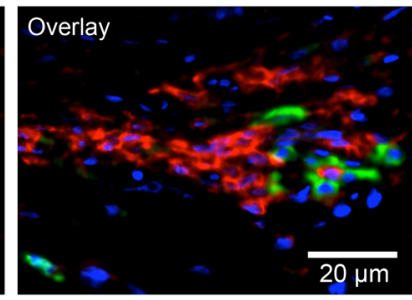

Figure 1. Quantification of T and B cells in SSc lesions. (A) Representative multicolor immunofluorescence image of CD3 ${ }^{+} T$ cells (red) and CD19+ $B$ cells (green) in an SSc lesion. (B) Absolute numbers per $\mathrm{mm}^{2}$ of $\mathrm{CD3}^{+} \mathrm{T}$ cells and CD19+ $\mathrm{B}$ cells in lesions from 35 patients with SSc. (C) Relative proportions of $\mathrm{CD}^{+}$and CD8 ${ }^{+} \mathrm{T}$ cells in skin lesions of individual SSc patients $(n=35)$. (D) Representative multicolor immunofluorescence images of CD4 ${ }^{+}(\mathrm{red})$ and $\mathrm{CD} 8^{+}$ (green) T cells in SSc tissues. Data are presented as mean \pm SEM. ${ }^{* * *} P<0.0001$ by Mann-Whitney $U$ test.

CD4 ${ }^{+}$CTLs in SSc to be increased in comparison with both healthy control skin and skin affected by bullous pemphigoid (Figure 2C and Supplemental Figure 2). When compared with healthy donor skin samples, $77 \%$ of the skin samples from SSc patients demonstrated an expansion of infiltrating CD $4^{+}$CTLs (using the healthy donor mean +2 SD as a cutoff) and in many of these patients, $\mathrm{CD} 4{ }^{+} \mathrm{CTL}$ were the dominant $\mathrm{CD} 4{ }^{+} \mathrm{T}$ cell subset identified at the site of disease (Figure 2B).

Other $\mathrm{CD}^{+} \mathrm{T}$ cell subsets have also been implicated in the pathogenesis of SSc but comprehensive and quantitative analyses of tissues have not been previously reported (11-14, 19). In order to more comprehensively quantify all major $\mathrm{CD} 4^{+} \mathrm{T}$ cell subsets, including Th1, Th2, Th17, and Tfh cells, as well as Tregs and CD $4^{+}$ CTLs, we selected tissues from a subset of our patients, including a sampling of subjects with $\mathrm{CD} 4^{+}$CTL numbers above, at, and below the median (Figure 2, D and E, and Supplemental Figure 3). In 2 of the 10 patients, Th17 cells were more prominent than $\mathrm{CD}^{+}$CTLs in terms of relative proportions (Figure 2E). However, in the remaining subjects, $\mathrm{CD} 4^{+} \mathrm{CTLs}$ represented the dominant tissue-infiltrating $\mathrm{CD} 4^{+} \mathrm{T}$ cell subset in SSc (Figure 2E). Representative images of markers used to define each $\mathrm{CD} 4^{+} \mathrm{T}$ cell subset are displayed in Supplemental Figure 3.

We previously demonstrated an expanded tissue infiltrate dominated by $\mathrm{CD} 4{ }^{+}$CTLs in patients with $\operatorname{IgG} 4-\mathrm{RD}(26,27)$. Those cells express multiple profibrotic and proinflammatory molecules at sites of disease. As in IgG4-RD, a subset of infiltrating $\mathrm{CD} 4^{+}$CTLs in SSc tissues was found to actively express IL-1 $\beta$ (Supplemental Figure 4, A and B).

Given the prominent contribution of $\mathrm{CD} 4{ }^{+}$CTLs to the overall $\mathrm{CD} 4^{+} \mathrm{T}$ cell infiltrate and because the numbers of $\mathrm{CD} 4^{+}$and $\mathrm{CD}^{+} \mathrm{T}$ cells infiltrating SSc tissues were comparable, we sought to determine whether $\mathrm{CD}^{+} \mathrm{T}$ cells in the tissues were potentially cytotoxic. Although on average a greater proportion of $\mathrm{CD}^{+}$ $\mathrm{T}$ cells (compared with $\mathrm{CD} 4^{+} \mathrm{T}$ cells) expressed GZMA, the absolute numbers of $\mathrm{GZMA}^{+} \mathrm{CD} 8^{+} \mathrm{T}$ cells were similar to those of $\mathrm{GZMA}^{+} \mathrm{CD} 4^{+} \mathrm{T}$ cells (Figure $3, \mathrm{~A}^{-} \mathrm{C}$ ). 
A CD4 GZMA GATA3 DAPI

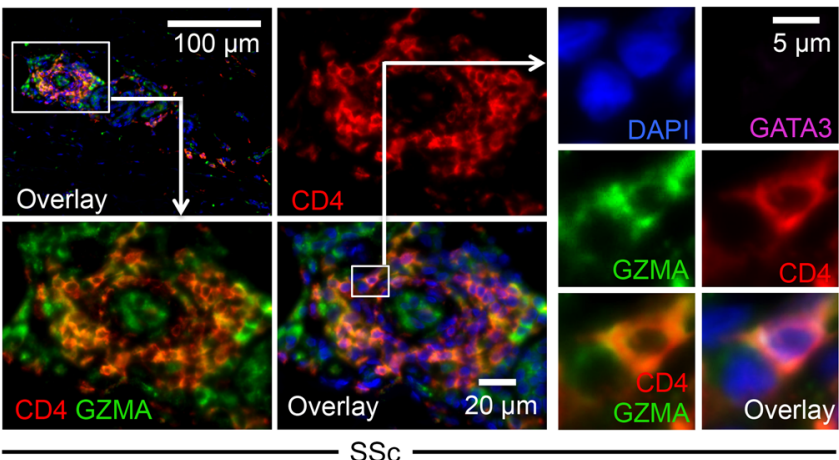

SSc

B

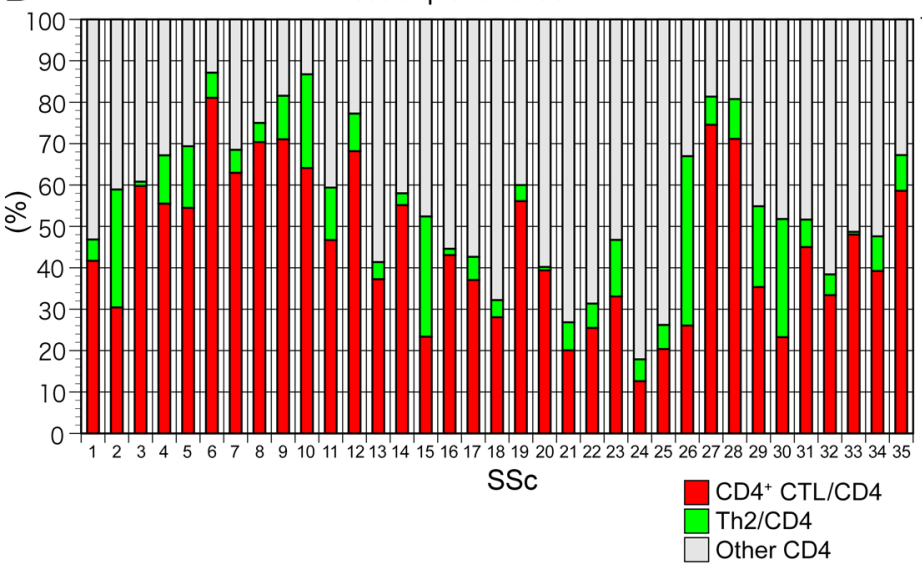

CD4 GZMA GATA3 DAPI
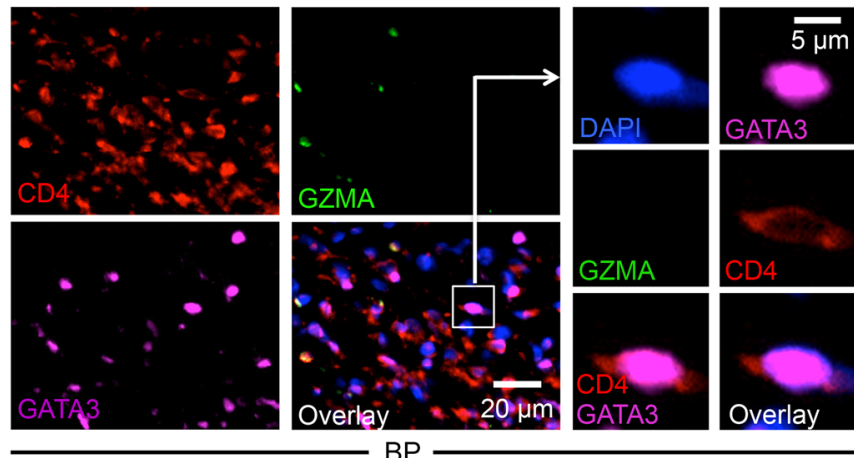

C

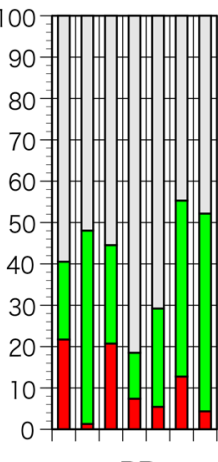

BP
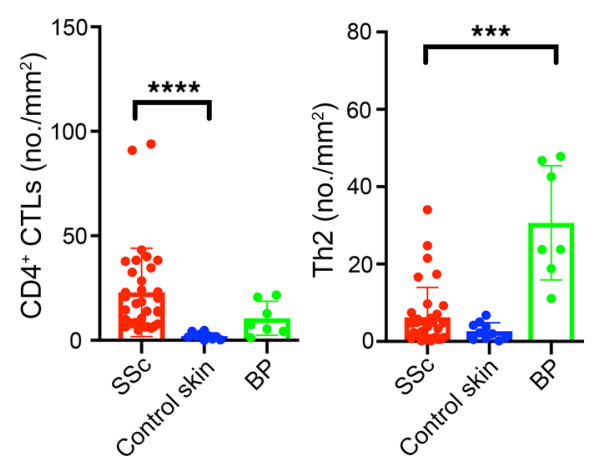

D

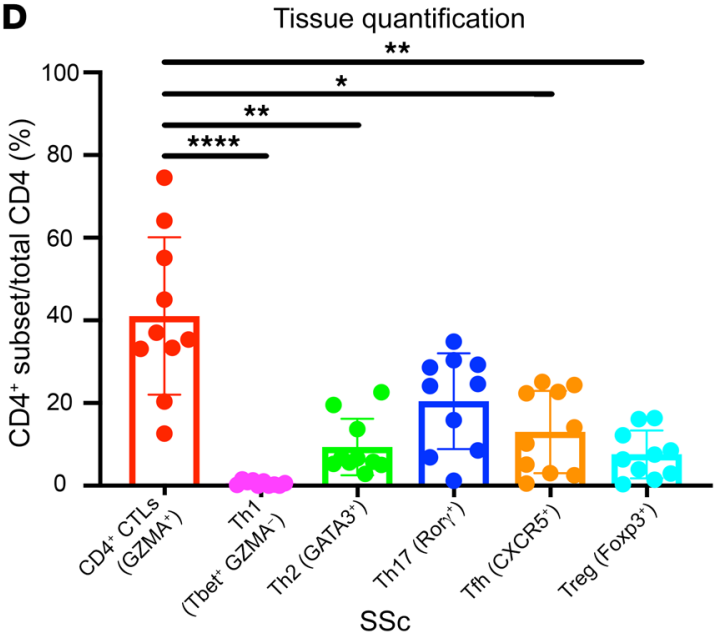

E Tissue quantification

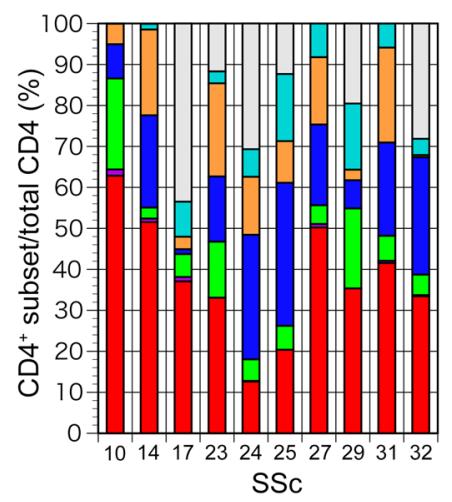

CD4 ${ }^{+}$CTL/CD4

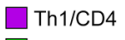

$\square$ Th2/CD4

$\square$ Th17/CD4

$\square$ Tfh/CD4

$\square$ Treg/CD4

$\square$ Other CD4

Figure 2. CD4+ CTLs are abundant in skin lesions of SSc patients. (A) Representative multicolor immunofluorescence image of cells coexpressing CD4 (red) and GZMA (green) that infiltrate the skin in SSc. The right panel additionally displays CATA3 (purple) staining to identify Th2 cells in bullous pemphigoid (BP) tissue. GATA3 staining was also undertaken in SSc. (B) Relative proportions of CD4+ CTLs (red), Th2 cells (green), and other CD4+ cells (gray) in SSc $(n=35)$ and BP $(n=7)$. (C) Absolute number of CD4+ CTLs and Th2 cells per $\mathrm{mm}^{2}$ of skin, comparing SSc $(n=35)$ to control skin $(n=10)$ samples and BP $(n=7)$. Multiple comparisons are controlled for by Kruskal-Wallis test. ( $D$ and $\mathbf{E})$ Relative proportions of Th1, Th2, Th17, and CD4+ CTL subsets in tissues from 10 SSc patients. Relative proportions of each subset (D) and of each subset in each patient (E) are depicted. Multiple comparisons are controlled for by Kruskal-Wallis test. Data are presented as mean \pm SEM. ${ }^{*} P<0.05 ;{ }^{* *} P<0.01 ;{ }^{* * *} P<0.001 ;{ }^{* * *} P<0.0001$.

Endothelial cells are frequent targets of apoptosis and upregulate HLA class II in SSc tissues. We previously observed the functional capacity for cytotoxicity by CD4+ CTLs in in vitro coculture experiments (26). To explore the possibility of the cytotoxic targeting of host cells by tissue-infiltrating cytotoxic T cells, we quantified the number of cells undergoing apoptotic cell death using an antibody that recognizes cleaved caspase-3 (cCasp-3) (Figure 4A). Confirming previous reports associating tissue apoptosis with the patho- 
A CD4 CD8 GZMA DAPI
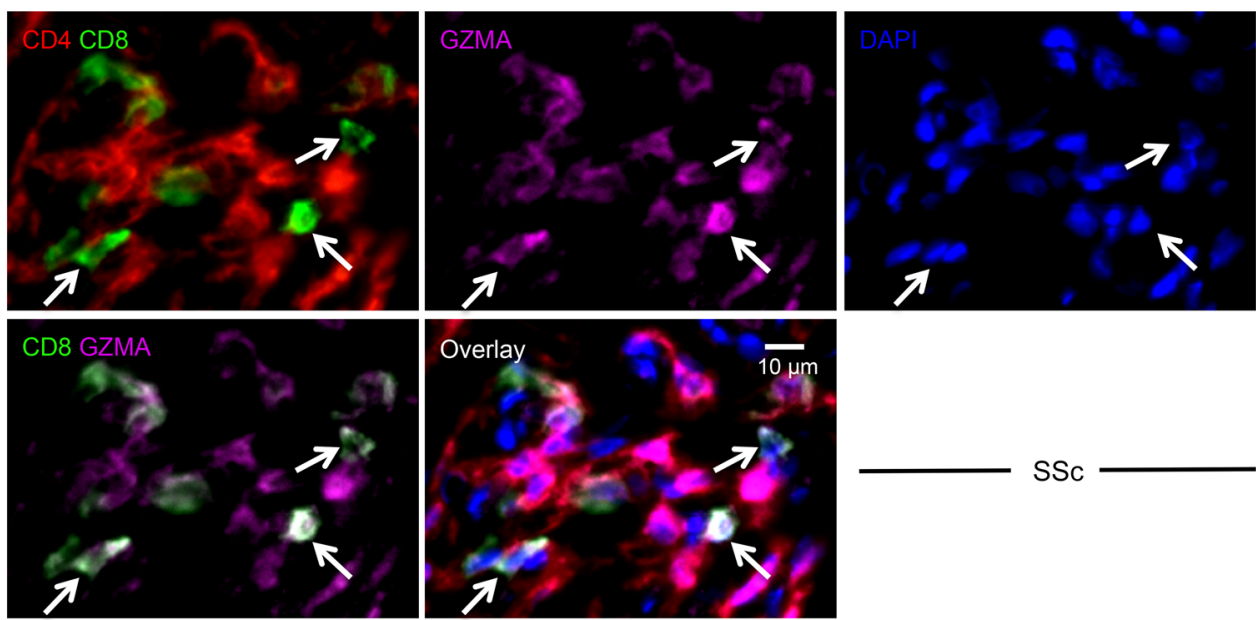

B Tissue quantification

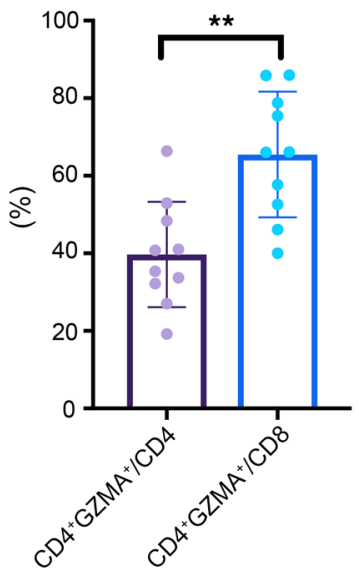

C Tissue quantification

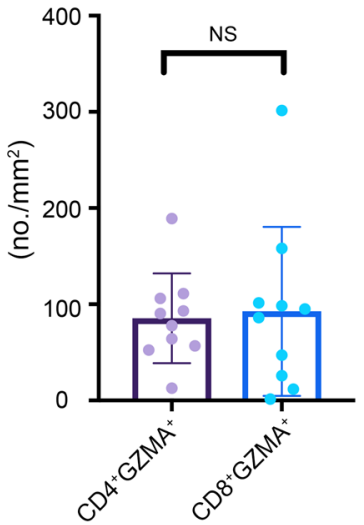

Figure 3. $\mathrm{CDB}^{+} \mathrm{CZMA}{ }^{+} \mathrm{T}$ cells infiltrate tissue in SSc. (A) Representative multicolor immunofluorescence images of CD8+ (green) GZMA+ (purple) T cells (delineated by arrows) in an SSc skin biopsy. (B) Relative proportions of CD4+ and $\mathrm{CD} 8^{+}$T cells expressing CZMA ( $n=$ 10). (C) Absolute numbers of CD4+ $\mathrm{CZMA}^{+}$and $\mathrm{CD}^{+} \mathrm{CZMA}^{+} \mathrm{T}$ cells in skin biopsies of 10 patients with SSc. Data are presented as mean \pm SEM. ${ }^{* *} P<$ 0.01 by Mann-Whitney $U$ test. genesis of SSc, we observed a marked increase in the absolute number of apoptotic cells in SSc tissues compared with those of controls (Figure 4, B and C). The numbers of apoptotic cells found in tissues affected by SSc were often quite prominent, in the range of 50 to 100 apoptotic cells per high-power field (Figure 4C).

Given the many previous reports describing, but not quantitating, endothelial cell apoptosis in SSc, we explored the origin of apoptotic cells in SSc tissues to provide a comprehensive tissue-based analysis and determine if endothelial cells were, in fact, prominent targets of apoptosis in this disease. We first costained for cCasp-3 and markers of major immune cell lineages and found that the majority of apoptotic cells did not express CD19, CD3, and CD68, indicating that very few macrophages and lymphoid cells undergo apoptosis in SSc skin (Figure 4D). Because observations such as nailfold capillary obliteration, digital ulceration, and renal crisis are all frequently associated with the vasculopathy of SSc but distinctly absent in the context of IgG4-RD, we used the latter disease as a source of control tissue samples to assess the preferential targeting of endothelial cells to undergo apoptosis in ex vivo tissue samples. Consistent with previous reports, we observed a striking and significant increase in the proportion of apoptotic cells accounted for by dying endothelial cells in SSc (Figure 5, A and B, and Supplemental Figure $5 \mathrm{~A}$ ). In some cases, more than $50 \%$ of all apoptotic cells in SSc tissues were of endothelial origin (Figure 5B). In addition, infiltrating $\mathrm{CD}^{+}{ }^{+}$CTLs were often observed enveloping endothelial cells (Figure 5C and Supplemental Figure 5B), with some of these associated endothelial cells undergoing apoptosis with GZMA visible within the cytosol, suggesting that endothelial cells may represent targets of $\mathrm{CD} 4^{+} \mathrm{CTL}$-directed cytotoxicity (Figure 5D).

Because $\mathrm{CD}^{+}$CTLs mediate cell death in an HLA class IIrestricted manner, we wondered if the endothelial cells in SSc may have upregulated HLA class II, permitting their recognition by HLA class II-restricted TCRs on CD4 ${ }^{+}$CTLs (38). We found a significant increase in HLA-DR expression on endothelial cells in SSc tissues compared with their counterparts in healthy control skin (Figure 5E). Some of the endothelial cells expressing HLA-DR were observed to contain cCasp-3, indirectly suggesting that antigen presentation by HLA class II, and possibly interactions with antigen-specific $\mathrm{CD} 4{ }^{+}$CTLs, may have contributed to their apoptotic death (Figure 5F and Supplemental Figure 5C). The relative efficiency with which we have detected apoptotic cells - which are generally cleared rapidly by macrophages - may in part be explained by the relative paucity of macrophages in SSc tissues, as seen in Supplemental Figure 6.

Analyses of $\mathrm{CD}^{+} \mathrm{CTL}$ heterogeneity define a specific subset relevant to SSc. To further characterize CD $4^{+}$CTLs in the disease- 

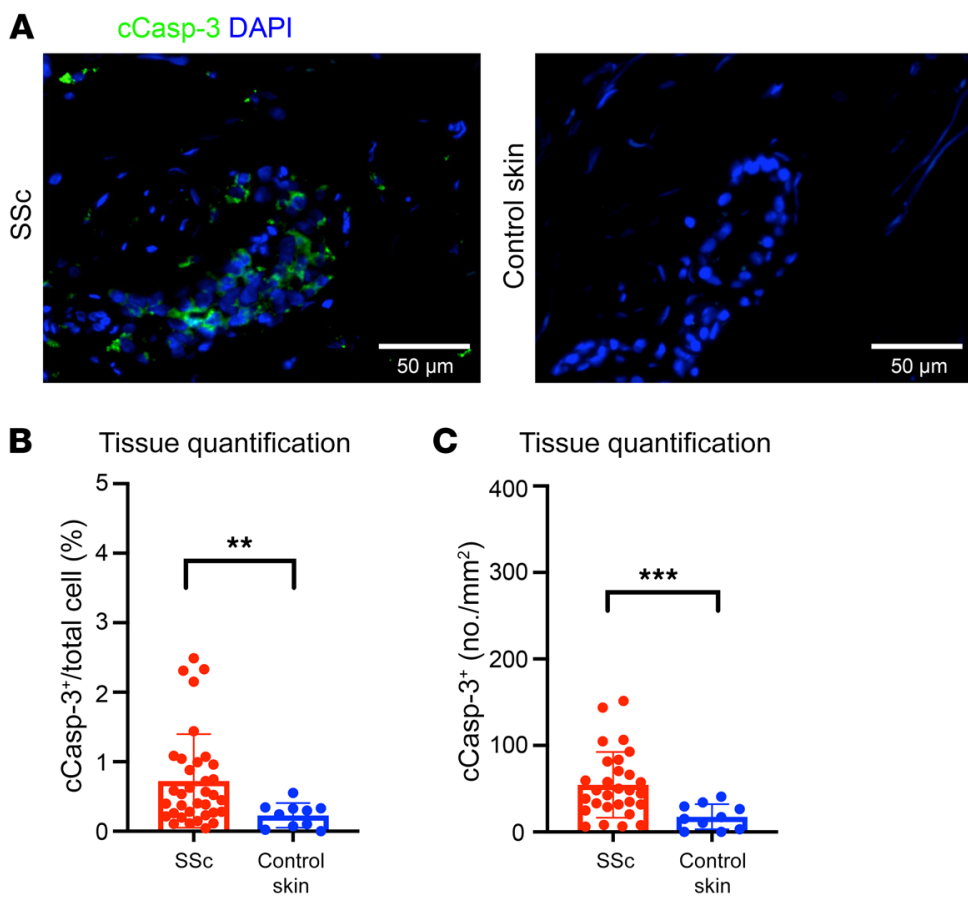

D

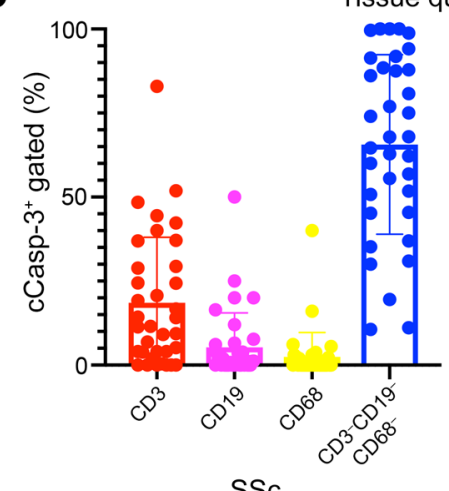

SSc

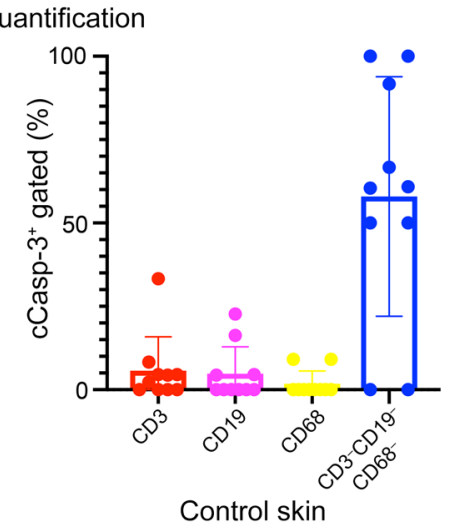

Figure 4. Apoptotic cells are frequently seen in SSc tissues. (A) Representative multicolor immunofluorescence images showing cleaved caspase-3 (cCasp-3) staining (green) in SSc and control skin samples. (B and C) Proportions (B) and absolute numbers (C) of cCasp- $3^{+}$apoptotic cells in SSc $(n=35)$ and control skin $(n=10)$. (D) Proportions of apoptotic cells in SSc $(n=35)$ and control skin $(n=10)$ accounted for by T cells (red), $B$ cells (purple), macrophages (yellow), and other cells (blue). Data are presented as mean $\pm \mathrm{SEM} .{ }^{* *} P<0.01,{ }^{* * *} P<0.001$ by Mann-Whitney $U$ test.

ing $\mathrm{CD} 57{ }^{\text {hi }} \mathrm{CD} 4^{+} \mathrm{CTL}$ to positively correlate with the extent of activated myofibroblasts within the paired skin samples (Figure 6C). Analyzing the SSc subjects with increased circulating $\mathrm{CD} 57^{\mathrm{hi}} \mathrm{CD} 4^{+}$CTLs compared to those with normal levels, we found a correlation with increasing age (Supplemental Figure 7B) but no correlation with gender, disease duration, auto-antibody profile, modified Rodnan skin score, the presence of digital ulceration, or the presence of renal crisis.

Because CD57hi CD $4^{+}$CTLs were composed of both $\mathrm{CD} 28^{\text {hi }}$ and $\mathrm{CD} 28^{\text {lo }}$ cells, and the $\mathrm{CD} 28^{\text {hi }} \mathrm{CD} 57^{\text {lo }}$ subset of $\mathrm{CD}^{+}$CTLs most often dominated the composition of $\mathrm{CD} 4^{+}$CTLs from healthy donors (Supplemental Figure 7A), we further phenotyped these respective subsets by additional surface and intracellular flow cytometry studies. Examining various effector molecules of cytotoxicity including GZMA, granzyme B (GZMB), and perforin (PRF), we found the $\mathrm{CD} 28^{\text {hi }} \mathrm{CD} 57^{\text {lo }} \mathrm{CD} 44^{+} \mathrm{CTL}$ to most closely resemble naive $\mathrm{CD} 4^{+} \mathrm{T}$ cells, $\mathrm{CD} 28^{\mathrm{lo}} \mathrm{CD} 57^{\mathrm{hi}} \mathrm{CD} 4^{+}$ CTLs to most closely resemble $\mathrm{CD} 8^{+} \mathrm{CTLs}$, and $\mathrm{CD} 28^{\text {hi }}$ CD57 ${ }^{\text {hi }} \mathrm{CD} 4^{+}$CTLs to have an intermediate phenotype (Figure 6D and Supplemental Figure 7C). Consistent with the idea of progressive differentiation from a precursor to an effector phenotype of $\mathrm{CD} 4^{+} \mathrm{CTLs}$, the CD28 $8^{\text {lo }}$ $\mathrm{CD} 57^{\text {hi }}$ subset expressed the greatest amount of CX3CR1, a marker previously associated with effector $\mathrm{CD} 4^{+} \mathrm{CTLS}$ and protective antiviral immunity, whereas the $\mathrm{CD} 28^{\text {hi }} \mathrm{CD} 57^{\text {lo }}$ subset expressed the greatest amount of CD127 (IL-7R), which was previously reported as a surface marker of precursor CD $4^{+}$CTLs (Figure 6D and refs. 34, 42). Focusing on the CD $4^{+}$CTL subset with the strongest effector phenotype, we performed TCR repertoire analysis on $\mathrm{CD} 28^{\text {lo }} \mathrm{CD} 57^{\text {hi }} \mathrm{CD} 4{ }^{+}$CTLs sorted from the blood of SSc patients and observed marked clonal expansion of effector CD4 ${ }^{+}$ CTLs compared with their naive $\mathrm{CD}^{+} \mathrm{T}$ cell counterparts, with 1 to 2 clones dominating the TCR repertoire and representing $50 \%$ to $75 \%$ of all sequence reads (Figure $6 \mathrm{E}$ ).

The transcriptome of $C D 28{ }^{l o} C D 57^{h i} C D 4^{+}$CTLs is enriched by genes relevant to cytotoxicity, metabolic activity, and tissue remodeling. Following the above observations supporting the notion that $\mathrm{CD}^{+}{ }^{+} \mathrm{SLAMF}^{+}$Tem cells that have lost CD28 and gained CD57 expression represent the most differentiated effector subset of CD $4^{+}$CTLs, we sought to characterize the transcriptional features of this cell population in order to make inferences about its functional role in the pathogenesis of SSc. We sorted $\mathrm{CD} 4^{+}$naive T cells and $\mathrm{CD} 28^{\mathrm{lo}} \mathrm{CD} 57^{\mathrm{hi}} \mathrm{CD} 4^{+}$CTLs from the blood of $3 \mathrm{SSc}$ patients and performed whole transcriptome RNA sequencing. We searched for 
A

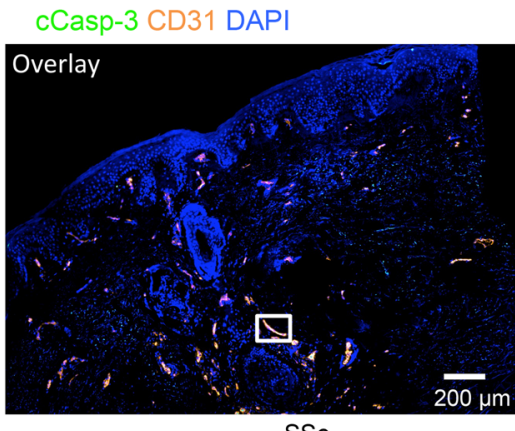

$\mathrm{SSc}$ c CD4 GZMA CD31 DAPI
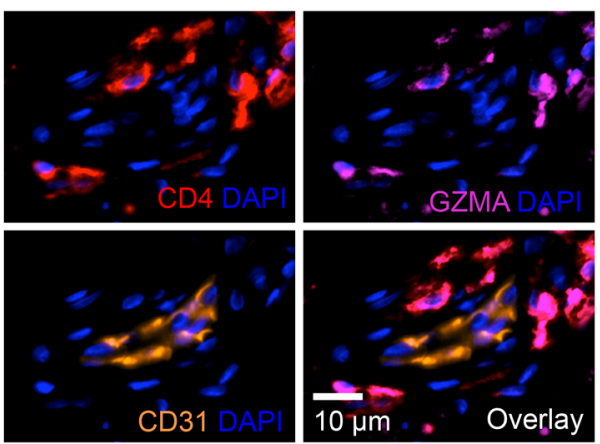

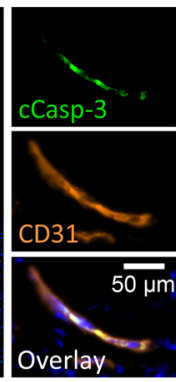

B

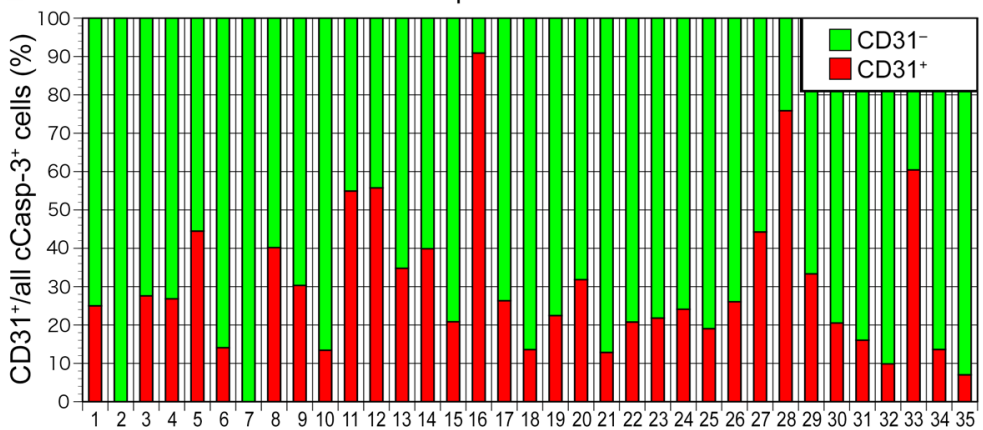

D CD4 GZMA cCasp-3 CD31 DAPI

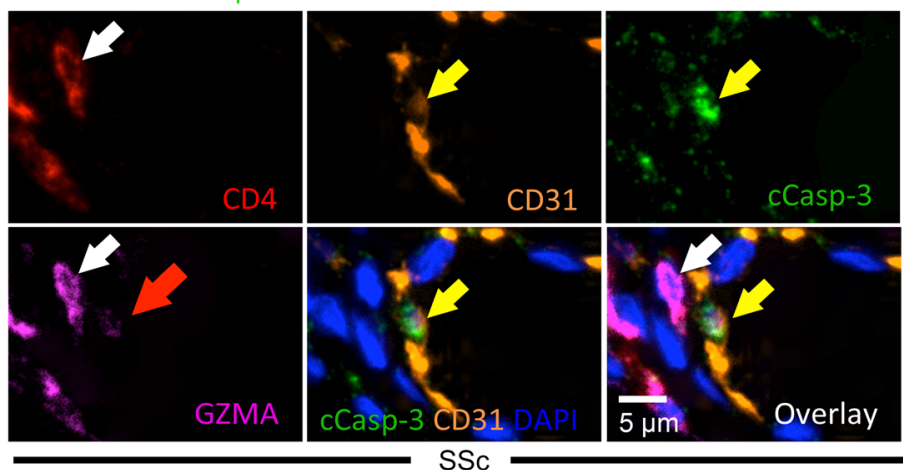

E

$\mathbf{F}$
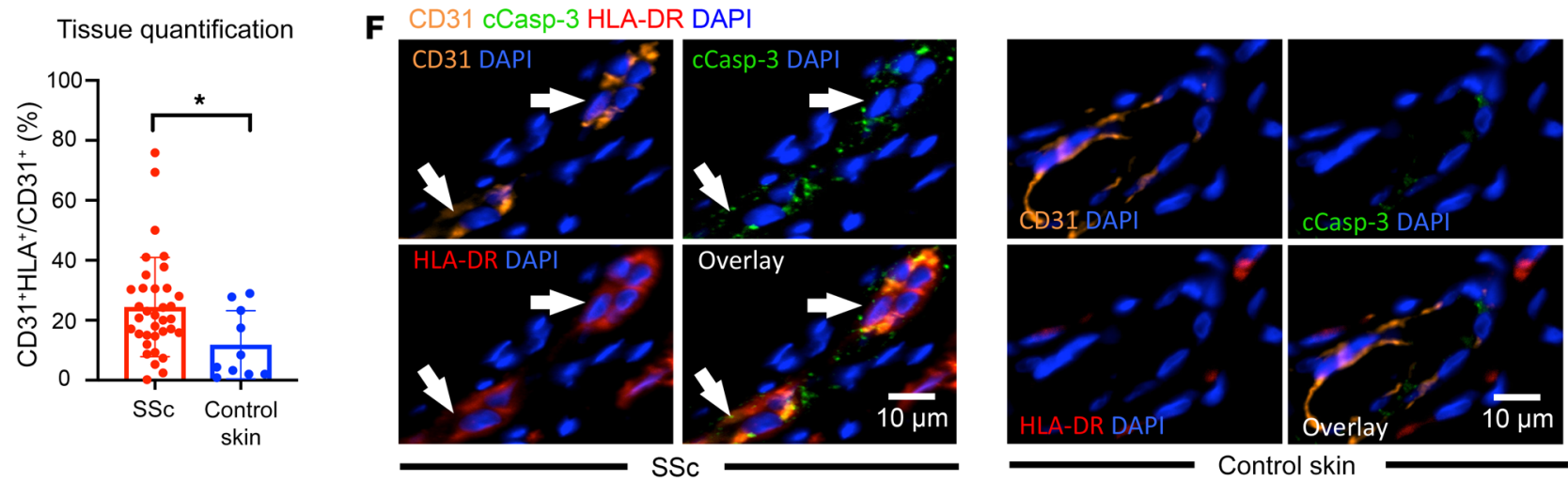

Figure 5. Endothelial cells expressing HLA class II are a frequent target of apoptosis in SSc tissues. (A) Representative multicolor immunofluorescence images showing CD31-expressing endothelial cells (orange) with cCasp-3 staining (green) in SSc. (B) Relative proportions of apoptotic cCasp-3+endothelial cells (red) and nonendothelial apoptotic cells (green) in SSc tissues $(n=35)$. (C) CD4 ${ }^{+}$(red) and CZMA ${ }^{+}$(purple) CD4+ CTLs accumulate in the vicinity of CD31+ (orange) endothelial cells in SSc tissues. (D) Representative multicolor immunofluorescence image showing a GZMA-expressing (purple) CD4+ CTL (white arrows) in close proximity to a cCasp-3+ endothelial cell (yellow arrow). Red arrow highlights GZMA visible within the apoptotic endothelial cell. (E) Percentages of $\mathrm{HLA}^{+}$cells among CD31+ cells in SSc $(n=35)$ and control skin $(n=10)$. (F) Multicolor immunofluorescence images of cCasp- $3^{+}(\mathrm{green})$ and $\mathrm{HLA}_{-\mathrm{DR}^{+}}$(red) endothelial cells (orange and indicated by arrows) in SSc, but not in control skin. Data are presented as mean $\pm \mathrm{SEM}$. ${ }^{*} P<0.05$ by Mann-Whitney $U$ test.

biologically meaningful transcriptional signatures using gene set enrichment analysis and compared effector $\mathrm{CD} 4^{+}$CTLs to naive $\mathrm{CD} 4^{+} \mathrm{T}$ cells in order to have the broadest comprehension of the $\mathrm{CD} 28^{\text {lo }} \mathrm{CD} 57^{\text {hi }} \mathrm{CD} 4^{+} \mathrm{CTL}$ transcriptome. As an exploratory analysis, we first used the CIBERSORT gene set database to suggest the cellular phenotype of the sorted $\mathrm{CD} 4^{+}$CTLs. In addition to gene sets available through CIBERSORT, we also included 2 curated gene sets representing the $\mathrm{CD} 4^{+} \mathrm{CTL}$ transcriptome based on available transcriptome data $(26,34)$. This validated our sorting strategy by confirming a cytotoxic phenotype of $\mathrm{CD} 28^{\mathrm{lo}} \mathrm{CD} 57^{\mathrm{hi}} \mathrm{CD} 4^{+}$CTLs, which are transcriptionally more similar to $\gamma \delta$ T cells and CD $8^{+}$CTLs than to traditional $\mathrm{CD} 4^{+} \mathrm{T}$ cells (Figure 7A). Based on the strongest enrichment observed with the $\mathrm{CD} 4^{+} \mathrm{CTL}$ curated gene lists, these cells have a distinctly different transcriptional signature compared with all other cytotoxic cell types, such as $\gamma \delta \mathrm{T}$ cells, $\mathrm{CD} 8^{+} \mathrm{CTLs}$, and NK cells, suggesting that they may play a unique biological role (Figure 7A). The cytotoxic transcriptional profile of $\mathrm{CD} 28^{\text {lo }} \mathrm{CD} 57^{\text {hi }} \mathrm{CD} 4^{+}$ CTLs includes upregulation of GZMA, GZMB, GZMH, GZMM, PRF1, GNLY, SLAMF7, CX3CR1, FGFBP2, NKG7, KLRB1, KLRD1, KLRF1, and KLRG1 (Figure 7B). Additionally, the upregulation of 
A

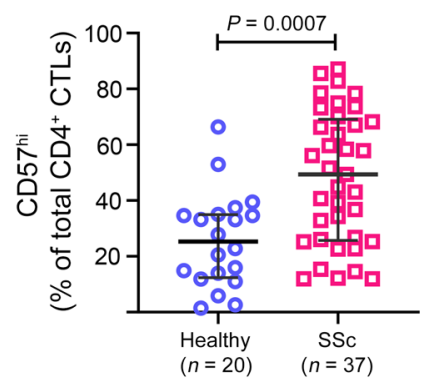

D

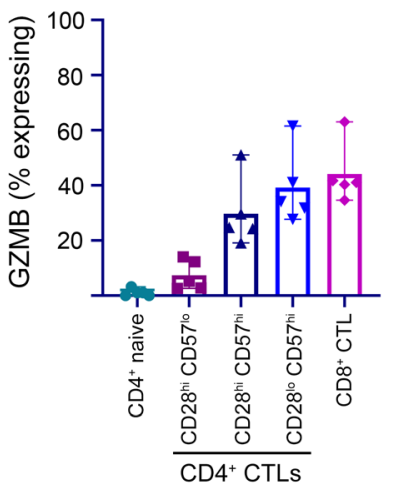

E

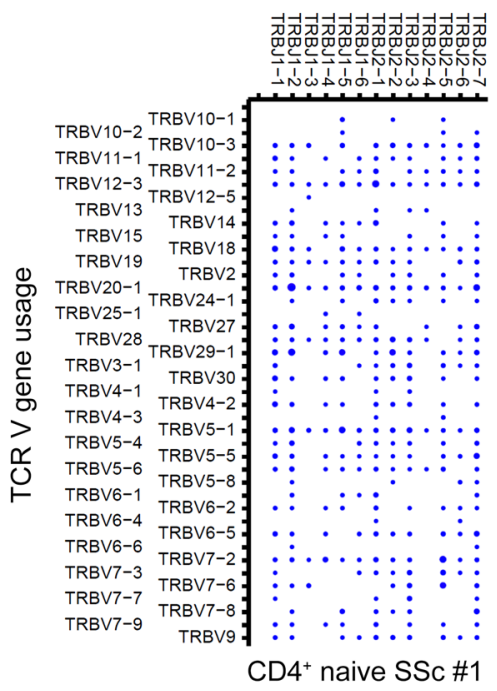

B

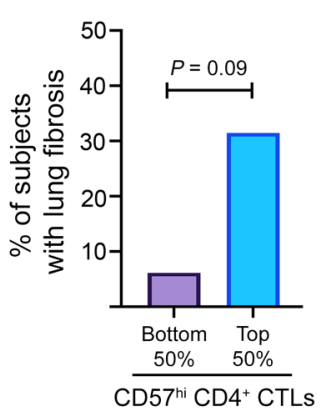

C $\mathrm{CD} 57^{\text {hi }} \mathrm{CD} 4^{+} \mathrm{CTL}$ expansion vs. skin fibrosis

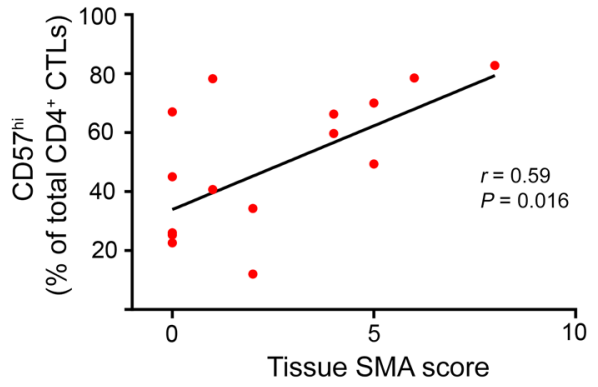

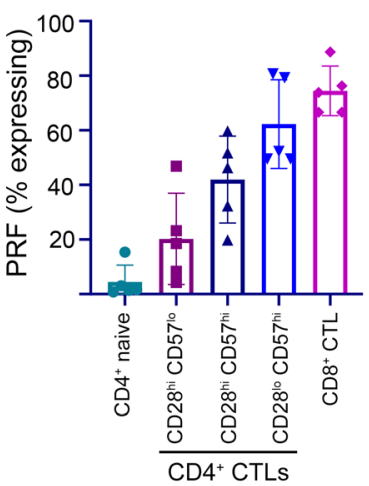

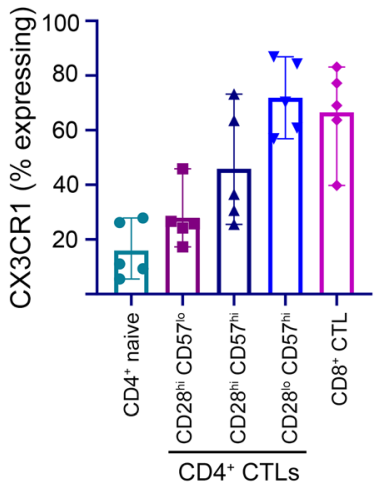

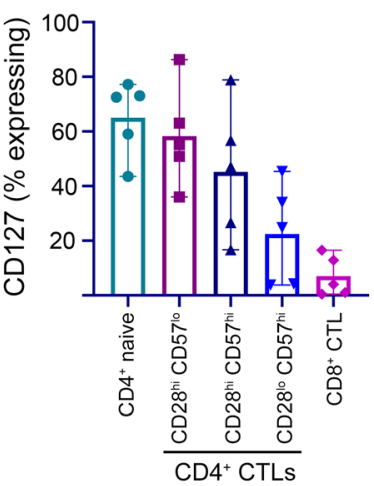

TCR J gene usage

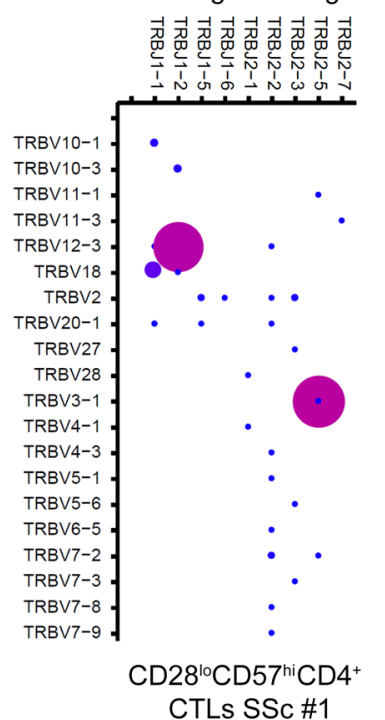

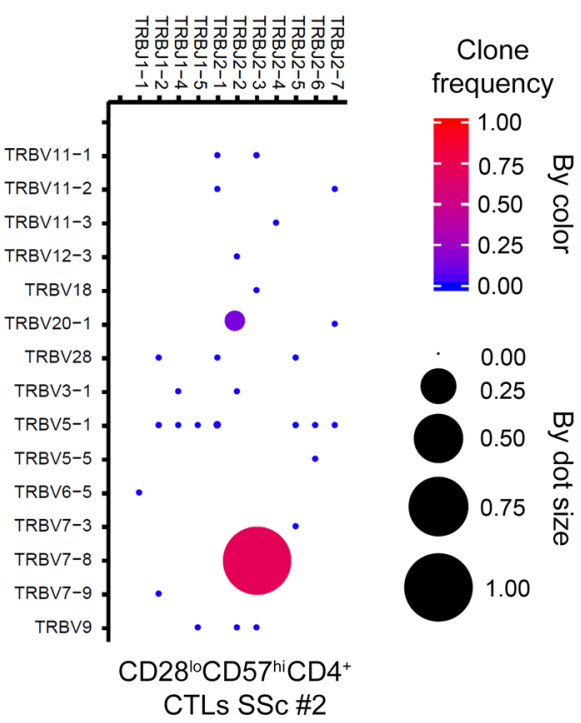

Figure 6. Effector CD4+ CTLs are clonally expanded and correlate with tissue fibrosis in SSc. (A) Dot plot demonstrates marked expansion of CD57 hicD4 ${ }^{+}$ CTLs comparing SSc subjects $(n=37)$ to age-matched healthy donors $(n=20)$. Lines represent medians with standard deviation. Mann-Whitney $U$ test was used to calculate $P$ value. (B) Bar chart showing increased frequency of lung fibrosis among SSc patients with greater CD57 hiCD4+ $C T L$ expansions. Proportion of patients in each group is plotted. $P$ value was calculated by Fisher's exact test. (C) X-Y plot showing positive correlation between CD57 ${ }^{\text {hicD }} 4^{+}$ CTL expansion and tissue $\alpha$-smooth muscle actin ( $\alpha-S M A)$ staining. Linear regression was used to calculate $P$ value. (D) Scatter plots with mean and range displaying the percentage of each T cell subset expressing granzyme B (GZMB), perforin (PRF), CX3CR1, and CD127. (E) Dot plots show the TCR repertoire comprising effector CD4 ${ }^{+}$CTLs from 2 SSc subjects (middle and right). TCR repertoire from a naive CD4 ${ }^{+}$T cell population is shown for reference (far left).

IL1B was observed in this CD4+ CTL subset, corroborating similar findings from the above tissue studies (Figure 7B).

Expanding upon our observations that $\mathrm{CD} 4^{+}$CTLs accumulate in both the vascular and tissue compartments of SSc patients, we curated a collection of gene sets relevant to tissue remodeling to understand if this cell population is enriched by relevant genes. These analyses revealed that gene expression in $\mathrm{CD} 28^{\mathrm{lo}} \mathrm{CD} 57^{\mathrm{hi}} \mathrm{CD} 4^{+}$ CTLs consistently enriched for gene sets relevant to tissue remodeling across multiple databases (Figure $7 \mathrm{C}$ ). Expression of genes such as PLG, FGA, KLKB1, and FN1 related to hemostasis; TGFB1, 
A
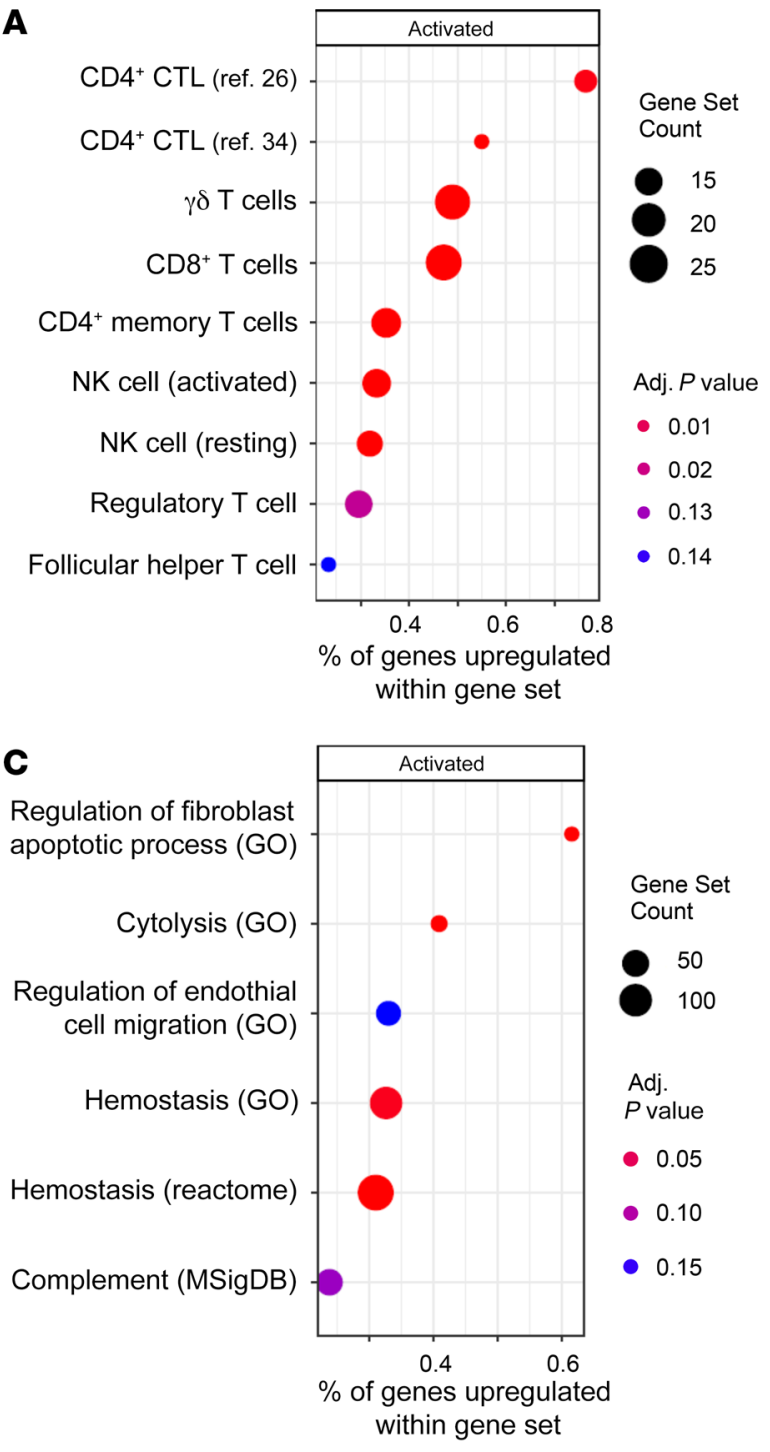

B
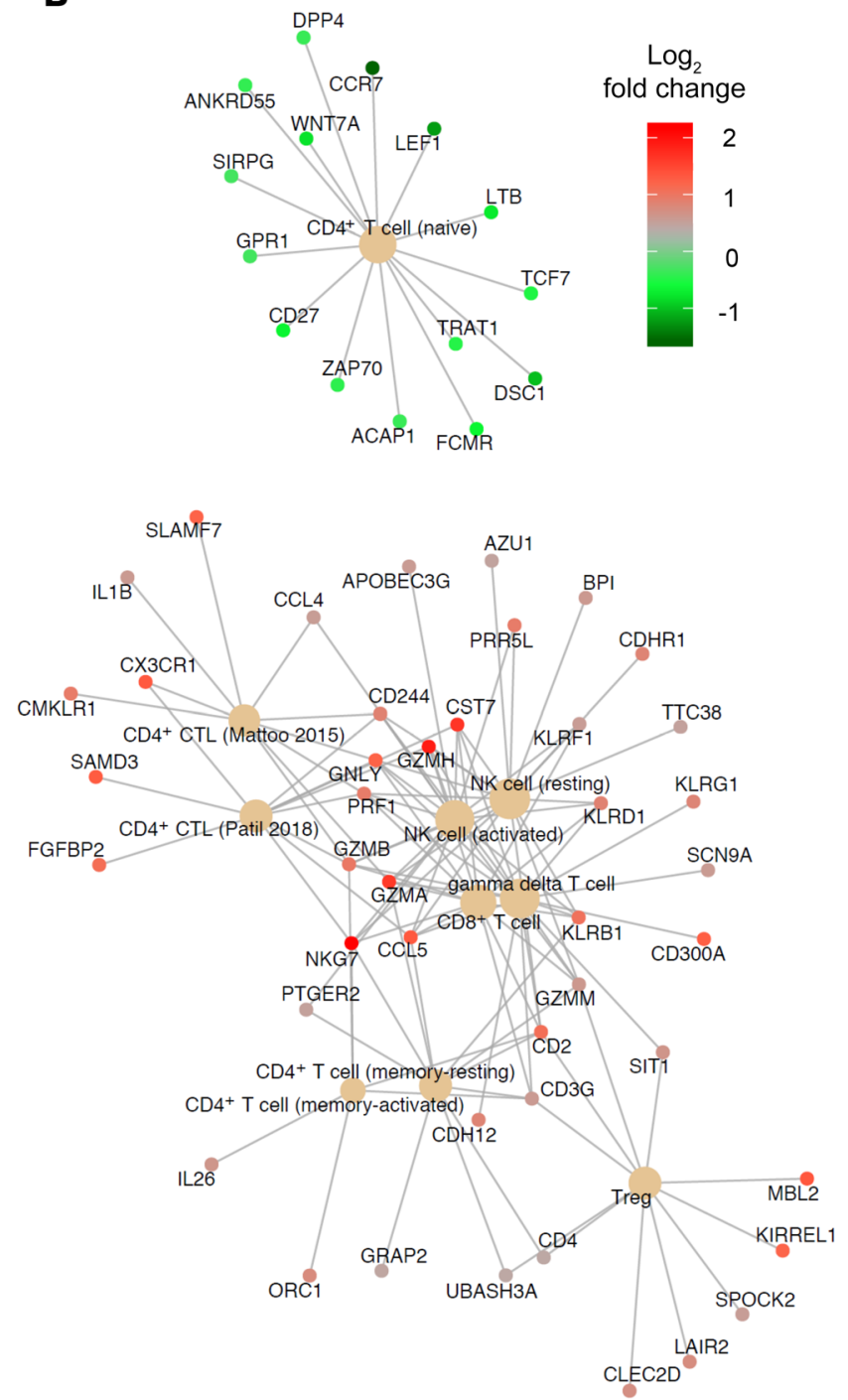

Figure 7. $\mathrm{CD} 28^{\text {lo }} \mathrm{CD} 57^{\text {hi }} \mathrm{CD} 4{ }^{+} \mathrm{CTLs}$ from SSc patients have transcriptional signatures linked to cytotoxicity, fibrogenesis, and metabolic activity rather than senescence. Comparing $\mathrm{CD} 28^{10} \mathrm{CD} 57^{\text {hi }} \mathrm{CD} 4^{+} \mathrm{CTLs}$ to naive $\mathrm{CD} 4^{+} \mathrm{T}$ cells, we identified enrichment for many gene sets, including (A) alignment with previously reported $C D 4^{+}$CTL transcriptomes and greater similarity with $\gamma \delta$ T cell and CD8 ${ }^{+} T$ cell transcriptomes rather than with conventional CD4 ${ }^{+} T$ cells, and (B) those associated with fibrogenesis such as regulation of fibroblast apoptosis, regulation of endothelial cell migration and hemostasis. Size of dots represents number of genes within a given gene set. The $x$ axes represent the percentages of genes within a gene set enriched for by the effector CD4+ CTL transcriptome. (C) CNETplot showing all genes upregulated by effector CD4+ CTLs from immune cell transcriptional gene sets showing a prominent cytotoxic profile with upregulation of GZMA, GZMB, GZMM, GZMH, PRF1, and SLAMF7. P values were calculated with the permutation method implemented in fgsea version 1.12.0.

TGFB3, PDGFB, and PDGFD related to fibrogenesis; and VEGFD and $P D G F$ (the latter also relevant to fibrogenesis) related to angiogenesis suggests that this subset of $\mathrm{CD} 4^{+} \mathrm{CTLs}$ may play a crucial role in tissue remodeling and fibrosis (Supplemental Figure 8).

To explore biological pathways that may be relevant to CD28 $8^{\text {lo }}$ $\mathrm{CD} 57^{\text {hi }} \mathrm{CD} 4{ }^{+}$CTLs, we examined enrichment among the Reactome gene set database. We found gene expression in $\mathrm{CD} 28^{\mathrm{lo}} \mathrm{CD} 57^{\mathrm{hi}} \mathrm{CD} 4^{+}$ CTLs to be linked to gene sets that suggest these cells are poised for survival (upregulated KRAS signaling, MYC target genes) and have a metabolically active phenotype (upregulated mTORC1, oxidative phosphorylation) (Supplemental Figure 9). We also observed transcriptomic evidence that $\mathrm{CD} 28^{\mathrm{lo}} \mathrm{CD} 57^{\mathrm{hi}} \mathrm{CD} 4{ }^{+} \mathrm{CTL}$ are conditioned by type I and type II interferons, both of which have been implicated in the pathogenesis of systemic sclerosis (43-45). Overall, these findings are consistent with the notion that $\mathrm{CD} 28^{\mathrm{lo}} \mathrm{CD} 57^{\mathrm{hi}} \mathrm{CD} 4^{+}$ CTLs represent an activated effector population of $\mathrm{CD}^{+} \mathrm{T}$ cells involved in the ongoing immune response at the time of sample collection rather than a resting memory or exhausted $\mathrm{T}$ cell population.

Ongoing $T$ cell activation may be a feature of early diffuse SSc. CTLA4-Ig (abatacept) has the ability to block the interaction of CD28 on naive T cells with CD80 and CD86 on antigen-presenting cells. The above blood studies from SSc patient samples suggest the progressive differentiation of $\mathrm{CD} 4{ }^{+} \mathrm{CTL}$ from a precursor population, to an intermediate subset marked by the gain of CD57 expres- 


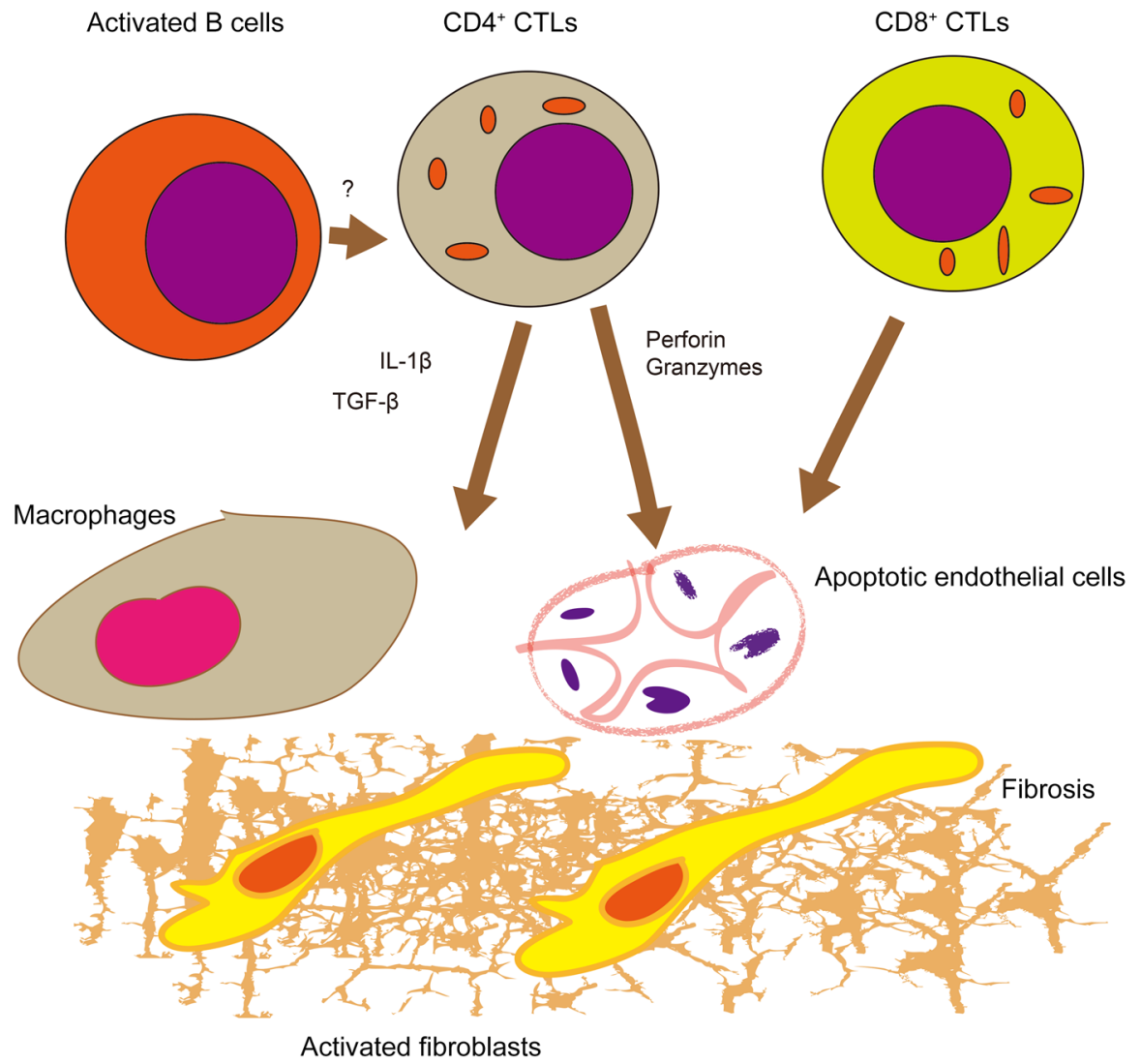

Figure 8. Schematic model for the pathogenesis of SSc. Infiltration of inflamed tissues by $\mathrm{CD}^{+}{ }^{+}$CTLs and $\mathrm{CD} 8^{+}$CTLs and the apoptosis of endothelial cells may contribute to this disease. Reactivation of $\mathrm{CD4} 4^{+} \mathrm{CTLs}$ may require antigen presentation by activated $B$ cells in tissue sites. Cell apoptosis and macrophage and fibroblast activation by cytokines secreted by cytotoxic $T$ cells may together contribute to fibrosis.

sion, and finally to a fully differentiated effector subset that has gained CD57 and lost CD28 expression. In addition to these two CD28-expressing CD4 ${ }^{+}$CTL subsets that may give rise to CD28 $8^{\text {lo }}$ $\mathrm{CD} 7^{\mathrm{hi}} \mathrm{CD} 4^{+} \mathrm{CTLs}$, effector $\mathrm{T}$ cells are likely being generated frequently in lymph nodes from naive or memory CD28-expressing $\mathrm{T}$ cells in the setting of ongoing disease activity following the presentation of self-antigens that have been captured by dendritic cells. In this context, it is plausible that CTLA4-Ig treatment may block the differentiation of naive $\mathrm{CD} 4^{+} \mathrm{T}$ cells, memory $\mathrm{CD} 4^{+} \mathrm{T}$ cells, and precursor subsets of $\mathrm{CD} 4^{+}$CTLs into bona fide effector $\mathrm{CD} 4^{+}$CTLs through interference with costimulation. To explore this, we examined pre- and posttreatment skin biopsies of 4 patients with early diffuse SSc who were enrolled in the ASSET trial examining the efficacy of CTLA4-Ig in SSc (46). We noted a discernible decline in tissue-infiltrating CD $4^{+}$CTLs in 3 of the 4 patients (Supplemental Figure 10). Ongoing T cell activation appears to be a feature of early diffuse SSc and there is therefore a rationale for targeting patients early in this disease with interventions that prevent $\mathrm{T}$ cell activation or attenuate $\mathrm{T}$ cell cytotoxicity.

\section{Discussion}

Using quantitative approaches to examine a range of infiltrating cells we have identified GZMA-expressing $\mathrm{CD} 8^{+} \mathrm{T}$ cells and $\mathrm{CD} 4^{+}$ CTLs as the most prominent T cells in SSc lesions. Quantitative analyses of immune cells in SSc tissues have not been undertaken previously, and our findings have attempted to elucidate the relevance of $\mathrm{T}$ cells, including several $\mathrm{CD} 4^{+} \mathrm{T}$ cell subsets, in this disease. Th2 and Tfh cells were found to infiltrate tissues only in low numbers in our studies. In contrast, $\mathrm{CD} 4^{+}$CTLs infiltrate SSc skin in high numbers, with expansions seen in nearly $80 \%$ of cases when compared with healthy donor tissues. In some subjects, an increase in Th17 cells was noted, but even in these biopsies the majority of infiltrating $\mathrm{T}$ cells are cells with the potential for cytotoxicity when considering both $\mathrm{CD} 4^{+} \mathrm{CTL}$ and $\mathrm{CD} 8^{+} \mathrm{T}$ cells.

Apart from inducing apoptosis, $\mathrm{CD} 4^{+} \mathrm{CTL}$ cells are known to secrete profibrotic as well as proinflammatory cytokines, including TGF- $\beta$ and IL-1 $\beta$. These cytokines may directly contribute to local tissue remodeling processes $(26,27)$. We observed the expression of IL-1 $\beta$ in CD $4^{+}$CTLs at the site of disease in SSc tissues, supporting a proinflammatory and/or profibrotic function of these cells in addition to a cytotoxic one.

Our current studies have identified a large number of apoptotic cells within the tissues of patients with SSc, concomitant with the accumulation of both $\mathrm{CD} 8^{+} \mathrm{CTLs}$ and $\mathrm{CD} 4^{+} \mathrm{CTLs}$, indirectly implicating these cytotoxic $\mathrm{T}$ cells in the targeted killing of host cells (26). We quantified the number of apoptotic cells in samples from patients, finding median values of approximately 100 cCasp $-3^{+}$cells per $\mathrm{mm}^{2}$ in SSc compared with control tissues, which had fewer than 15 apoptotic cells per $\mathrm{mm}^{2}$. Consistent with the prominent vasculopathy that precedes and then progresses concurrently with tissue fibrosis in SSc, we found endothelial cells to be a frequent target of apoptosis in SSc biopsies. Moreover, many endothelial cells in the SSc skin samples expressed HLA class II molecules. Collectively, these findings support the view that $\mathrm{CD}^{+} \mathrm{CTLs}$ and $\mathrm{CD} 8^{+} \mathrm{T}$ cells in SSc may contribute to vasculopathy and tissue fibrosis by targeted endothelial cell killing and by the elaboration of proinflammatory molecules such as IL-1 $\beta$, leading to overly exuberant tissue remodeling. 
Apoptosis of endothelial cells in SSc tissues has been noted for decades (47). Although anti-endothelial cell antibodies capable of inducing apoptosis of endothelial cells have been frequently reported, tissue studies precisely cataloguing the cell types undergoing apoptosis, or quantifying apoptotic cells have not previously been undertaken $\left(48^{-52}\right)$. We have observed that endothelial cells undergoing apoptosis are often in close proximity to cytotoxic $\mathrm{T}$ cells and sometimes can even be seen to have acquired GZMA in the cytosol. Future studies involving multicolor confocal microscopy on thick frozen sections of SSc biopsies and the use of cell distance mapping may provide further correlations about the potential physical interactions between cytotoxic $\mathrm{T}$ cells and endothelial cells in this disease.

The definitive identification of self-antigens that are recognized by expanded $\mathrm{T}$ cell clones that infiltrate tissues in SSc patients would strengthen the view that self-reactive $T$ cells drive this disease process. It is likely that certain relevant host proteins expressed at relatively high levels in endothelial cells contribute peptides that are presented on HLA class I molecules and that a distinct or overlapping subset of proteins yields peptides that are presented by HLA class II molecules. These antigens may come into play following a break in peripheral $\mathrm{T}$ cell tolerance and they presumably initiate immune responses in draining lymph nodes. Activated effector $\mathrm{CD}^{+}$and $\mathrm{CD}^{+}$cytotoxic $\mathrm{T}$ cells may then target host cells, particularly endothelial cells, in SSc tissues. The centromeric CENP-A and CENP-B proteins and the interferoninducible 16 protein (IFI-16), known antigens recognized by auto-antibodies in SSc, have been found to be expressed at relatively high levels in endothelial cells (53). Whether or not peptides from these antigens are presented on HLA molecules for $\mathrm{T}$ cell recognition and thus contribute to $\mathrm{T}$ cell-mediated endothelial cell apoptosis remains to be established.

Our studies quantitatively establish that the accumulation in the skin of $\mathrm{CD} 4^{+}$CTLs and $\mathrm{CD} 8^{+}$CTLs is a hallmark of early diffuse Ssc and suggest that similar quantitative tissue studies should be undertaken in other inflammatory diseases of the skin, including disorders in which fibrosis is not a dominant feature. We have previously noted that $\mathrm{CD} 4^{+} \mathrm{CTLs}$ accumulate in affected tissues in another fibrotic disease, IgG4-RD, but not in 2 nonfibrotic disorders, chronic sialadenitis and Sjögren's syndrome (26, 27). Although our results provide a potential mechanism for the long-described phenomenon of endothelial cell apoptosis in this disease, we also note that there is a general increase in apoptosis even in nonendothelial cells in SSc biopsies in comparison with normal skin. Although fibrosis is generally considered to be linked to inflammatory cytokines, apoptotic cells have been shown to trigger TAM family kinases on macrophages and thus accelerate wound healing (54). Apoptotic death in the lung resulting from germline mutations has also been postulated to contribute to the fibrotic process in idiopathic pulmonary fibrosis (55). Our data support the view that cytotoxic $\mathrm{T}$ cells may contribute to a fibrotic disease by both inducing apoptosis and secreting profibrotic cytokines. An overview model for the pathogenesis of SSc based on our observations is presented in Figure 8.

It is likely that in active SSc, there is ongoing presentation of antigen to naive $\mathrm{CD} 4^{+}$and $\mathrm{CD} 8^{+} \mathrm{T}$ cells and the recurrent activation of these cells in secondary lymphoid organs. The decline in
CD $4^{+}$CTLs after treatment with CTLA-Ig is consistent with such a view. It is therefore likely that interventions targeting cytotoxic $\mathrm{T}$ cells early in this disease may potentially halt the progression of this therapeutically intractable disease.

\section{Methods}

\section{Patient cohorts}

Skin tissue $(n=35)$ and blood $(n=28)$ samples from SSc subjects and skin tissue samples from healthy donors $(n=10)$ were obtained through a collaboration with the NIH-funded Autoimmune Center for Excellence at the University of Michigan. In addition, skin tissue samples from patients with bullous pemphigoid $(n=7)$ were obtained from the Department of Dermatology at the University of Pennsylvania. Twentysix of 28 blood samples were from patients with early diffuse SSc, whereas 1 had a limited phenotype and 1 was subsequently found to have scleroderma. Eighteen of these 28 blood samples and all tissue samples were collected from patients who were enrolled in ASSET, a placebo-controlled trial of abatacept versus placebo in patients with early dcSSc (45). All 28 blood samples and 35 skin tissue samples were obtained before the start of any immunosuppressive treatment. An additional 9 SSc subjects were recruited for blood sampling through the Massachusetts General Hospital Division of Rheumatology. Three of these 9 subjects had diffuse skin involvement and 6 were of the limited phenotype. Seven of these patients were not on any immunosuppression before sample collection, while the other 2 were on some form of treatment (1 on azathioprine $125 \mathrm{mg}$ daily and the other on leflunomide $20 \mathrm{mg}$ daily). Age-matched healthy donors $(n=20)$ were obtained through the Massachusetts General Hospital Division of Rheumatology. Healthy donors were defined by lacking any current or prior history of malignancy, autoimmune disease, or recurrent/chronic infections. Data such as sex, age, treatment status, disease activity, clinical manifestations, and laboratory parameters were extracted from the medical records of all patients used for these studies and are displayed in Supplemental Table 1. PBMCs were isolated by Ficoll within 4 hours of phlebotomy and preserved in liquid nitrogen until the time of use.

\section{Flow cytometry}

Frozen PBMCs were thawed and washed in complete DMEM. Before antibody staining, Fc receptors were blocked using Human TruStain FcX (BioLegend, 422302) at a dilution of 1:20 on ice for 15 minutes. Cells were stained for 20 minutes on ice, protected from light, at a concentration of 20 million cells/mL using the following antibody panel (manufacturer, clone, concentration used): anti-human CD3-BUV395 (BD Biosciences, clone SK7, 1:40), anti-human CD57-PE (BD Biosciences, clone NK-1, 1:400), anti-human CD8-BV785 (BioLegend, clone SK1, 1:300), anti-human CD45RA-BV605 (BioLegend, clone HI100, 1:300), antiCD4-BUV805 (BD Biosciences, clone SK3, 1:80), anti-human CD28BV480 (BD Biosciences, clone CD28.2, 1:40), anti-human CCR7-APC/ Cy7 (BioLegend, clone G043H7, 1:20), and anti-human SLAMF7-A648 (BD Biosciences, clone 235614, 1:10). Cells were washed with 1\% BSA in PBS after staining, centrifuged, and resuspended in 1\% BSA in PBS. Just before flow cytometry, dead cells were stained with SYTOX AADvanced Dead Cell Stain (Thermo Fisher Scientific, S10274). The following antibodies were used for intracellular flow cytometry studies: anti-human GZMA-PE/Cy7 (BioLegend, clone CB9, 1:10), anti-human GZMBBV421 (BD Biosciences, clone GB11, 1:25), anti-human GZMK-FITC 
(BioLegend, clone GM26E7, 1:25), and anti-human PRF-BV711 (BioLegend, clone dG9, 1:25). Anti-human CX3CR1-BV650 (BioLegend, clone 2A9-1, 1:25) was also used for extracellular staining before intracellular staining. Cells were fixed and permeabilized using the fixation/permeabilization solution kit from BD Biosciences (catalog 554714) according to the manufacturer's protocol. Flow cytometry was performed on a BD LSR II (BD Biosciences) and rainbow tracking beads were used to ensure consistent signals between flow cytometry batches. FCS files were analyzed using FlowJo software (version 10).

\section{Multicolor immunofluorescence staining}

Tissue samples were fixed in formalin, embedded in paraffin, and sectioned. These specimens were incubated with the following antibodies: anti-CD3 (catalog A045229-2, DAKO), anti-CD4 (catalog CM153A, Biocare), anti-CD19 (catalog CM310 A, B; Biocare), CD8 (catalog ab85792, Abcam), CD31 (catalog 3528; Cell Signaling Technology), CD68 (catalog ab955, Abcam), cleaved caspase-3 (catalog 9664, Cell Signaling Technology), GATA3 (catalog CM405A, Biocare), GZMA (catalog LS-C312742, LSBio), ROR $\gamma$ (catalog ab212496, Abcam), CXCR5 (catalog MAB190, R\&D Systems), Foxp3 (catalog 98377, Cell Signaling Technology), HLA-DR (catalog ab20181, Abcam), IL-1 $\beta$ (catalog MAB201, R\&D Systems), and SLAMF7 (catalog HPA055945, SigmaAldrich) followed by incubation with secondary antibody using an Opal Multiplex Kit (PerkinElmer). The samples were mounted with ProLong Diamond Antifade mountant containing DAPI (Invitrogen). In the analysis of apoptotic endothelial cells, we included only the tissue samples with sufficient numbers of apoptotic cells present. Relevant control tissues (healthy control skin for SSc) were used to determine sufficient apoptotic cell numbers based on the control median +1 SD. $\alpha$-Smooth muscle actin ( $\alpha$-SMA) tissue staining was performed, analyzed, and scored using a $10-\mathrm{cm}$ visual analog scale, as previously described (56).

\section{Microscopy and quantitative image analysis}

Images of the tissue specimens were acquired using the TissueFAXS platform (TissueGnostics). For quantitative analysis, the entire area of the tissue was acquired as digital grayscale images in 5 channels with filter settings for FITC, Cy3, Cy5, and AF75 in addition to DAPI. Cells of a given phenotype were identified and quantitated using the TissueQuest software (TissueGnostics), with cutoff values determined relative to the positive controls. This microscopy-based multicolor tissue cytometry software permits multicolor analysis of single cells within tissue sections similarly to flow cytometry. The principle of the method and the algorithms used have been described in detail elsewhere (57). Myofibroblast activation was graded within tissues by the $\alpha$-SMA score, as previously described (56).

\section{FACS isolation, bulk RNA sequencing, and TCR repertoire studies}

FACS isolation. Frozen PBMCs were thawed, washed, and stained with the same antibody panel as used for flow cytometry experiments, detailed above. Consistent with our flow cytometry studies, effector $\mathrm{CD}^{+}$CTLs were sorted based on the following gates: $\mathrm{CD}^{+}$, $\mathrm{CD}^{+}, \mathrm{CD}^{-}, \mathrm{CCR}^{-}, \mathrm{CD}^{2} 5 \mathrm{RA}^{-}, \mathrm{SLAMF}^{+}, \mathrm{CD} 28^{\text {lo }}$, and $\mathrm{CD} 57^{\text {hi }} . \mathrm{CD}^{+}$ naive $\mathrm{T}$ cells were sorted based on the following gates: $\mathrm{CD}^{+}, \mathrm{CD}^{+}$, $\mathrm{CD}^{-}, \mathrm{CCR}^{+}$, and CD45RA ${ }^{+}$. Cells were sorted directly into $350 \mu \mathrm{L}$ RLT-BME lysis buffer, vortexed briefly, and immediately frozen on dry ice. Cells were sorted on a BD FACSAria II with FACSDiva version 7. RNA was isolated from sorted cell lysates using RNeasy Plus
Micro Kits (Qiagen, 74034). For bulk RNA sequencing, isolated RNA was immediately processed through RT-PCR, as previously described (58). For TCR repertoire, isolated RNA was immediately processed through 5'RACE RT-PCR, as previously described $(59,60)$. Sequence data were deposited into the NCBI's Gene Expression Omnibus (GEO) repository with accession number GSE142663.

Bulk RNA sequencing. Raw FASTA files (read 1 and read 2) were processed with the zUMIs v2.2 pipeline using the splice-aware aligner STAR v2.5.4a, SAMtools v1.9, and pigz v2.3.4 (61). In short, reads were filtered according a predefined list matching the custom-made well barcodes in the oligo-dTs and unique molecular identifiers were then collapsed. The remaining reads were mapped to the reference genome with the use of STAR aligner. Exon counts from mapped reads were imported into R studio v1.1.456 and further processed for statistical analysis with DESeq2 v1.22.1. Because we compared multiple cellular subtypes per patient, we controlled for between-patient variability in the design matrix. Fitting of the models' dispersion to the mean intensity was performed with local regression and the effect sizes were calculated with the postcounts estimator in DESeq. Dispersion plot fits were visually validated for goodness of fit. We performed pairwise comparison between cell subtypes of interest and the per-gene $P$ values were adjusted for multiple testing by Benjamini-Hochberg ( $\mathrm{BH})$ false discovery rate (FDR). Gene-set enrichment analysis was done with the GSEABase package v1.44.0 (fgsea algorithm) with 10,000 permutations, geneset size between 5 and 2,000, and a BH-FDR-adjusted $P$ value cutoff of 0.25. Gene-set lists were downloaded from the MSigDB v6.2 at the Broad Institute of MIT \& Harvard and included gene sets from the GO, KEGG, BioCarta, Reactome, and Hallmark databases. Custom gene sets were created by extraction of the cell-type-specific gene expression signatures from Stanford's CIBERSORT tool and previous studies describing gene expression signatures on CD4 ${ }^{+}$CTLs $(26,34,62)$.

TCR repertoire analysis. Processed files were imported in VDJtools v1.1.4 for statistical analysis. Basic V-J usage per cell type was analyzed by computing read frequencies. Clonotype abundances were calculated by CDR3 nucleotide, $\mathrm{V}$ and J matching among CD $4^{+} \mathrm{CTLs}$.

\section{Statistics}

Flow cytometry, clinical correlations, and tissue studies. GraphPad Prism version 8 was used for statistical analysis, curve fitting, and linear regression. A 2-tailed Mann-Whitney $U$ test was used to calculate $P$ values for continuous, nonparametric variables. For categorical variables, Fisher's exact test was used to calculate $P$ values. For comparing more than 1 population, Kruskal-Wallis testing was used with Dunn's multiple-comparisons testing. Pearson's correlation coefficient was used to quantify the strength of linear relationship between variables. A $P$ value of less than 0.05 was considered significant.

\section{Study approval}

These studies were approved by the Institutional Review Boards at the Massachusetts General Hospital, University of Michigan Medical Center, Hospital of the University of Pennsylvania, and Kyushu University Hospital. All patients provided written informed consent before inclusion in the study.

\section{Author contributions}

TM and NK performed tissue analyses using multicolor immunofluorescence and quantitation of cellular infiltrates and apoptosis. 
CAP and HL performed flow cytometry and analyses of T cell subsets in blood, repertoire analyses, flow sorting, and RNA sequencing library preparation. HM contributed to repertoire studies with SJHM. HAC contributed to RNA sequencing studies along with JK. JK analyzed the RNA sequencing data. VSM designed the repertoire studies and contributed to all sequencing analyses. MG contributed to the statistical analyses. SSc tissues were provided by DK and RL. IgG4-RD tissues were provided by TM. Bullous pemphigoid tissues were provided by ASP. Clinical information on SSc patients was provided by DK. Blood samples were provided by DK, DF, and JHS. CAP and SP wrote the manuscript. All authors provided inputs. The studies were conceived by SP, HM, TM, CAP and NK. SP was responsible for the overall execution of the study.

\section{Acknowledgments}

We thank Candace Gregg for assistance in organizing the manuscript. This work was supported by the NIH Autoimmune Cen- ters of Excellence funding to SP, DK, and DF including U19 AI 110495 to SP and UM1 AI110498 to DK and DF. CAP was supported by a Rheumatology Research Foundation Scientist Development Award and a Sponsored Research Agreement with Union Chimique Belge (UCB). TM was supported by JSPS JP18KK0260 and the Takeda Science Foundation. JK was supported by a Work Visit Grant of the Amsterdam Infection and Immunity Institute. VSM was supported by AI113163 from the NIH. MG was supported by NIAID P30AI060354. ASP was supported in part by National Institute of Arthritis and Musculoskeletal and Skin Diseases (NIAMS) grant P30-AR06958. The contents are solely the responsibility of the authors and do not necessarily represent the official views of the NIAMS or the NIH.

Address correspondence to: Shiv Pillai, Ragon Institute, 400 Technology Square, Cambridge, Massachusetts 02139, USA. Phone: 857.268.7005; Email: pillai@helix.mgh.harvard.edu.
1. Denton CP, Khanna D. Systemic sclerosis. Lancet. 2017;390(10103):1685-1699.

2. Chora I, et al. Evidence for a derangement of the microvascular system in patients with a very early diagnosis of systemic sclerosis. J Rheumatol. 2017;44(8):1190-1197.

3. Mostmans Y, et al. The role of endothelial cells in the vasculopathy of systemic sclerosis: A systematic review. Autoimmun Rev. 2017;16(8):774-786.

4. Asano Y, Sato S. Vasculopathy in scleroderma. Semin Immunopathol. 2015;37(5):489-500.

5 . Morisset J, et al. Mortality risk prediction in scleroderma-related interstitial lung disease: The SADL model. Chest. 2017;152(5):999-1007.

6. Sullivan KM, et al. Myeloablative autologous stem-cell transplantation for severe scleroderma. N Engl JMed. 2018;378(1):35-47.

7. Burt RK, et al. Autologous non-myeloablative haemopoietic stem-cell transplantation compared with pulse cyclophosphamide once per month for systemic sclerosis (ASSIST): an open-label, randomised phase 2 trial. Lancet. 2011;378(9790):498-506.

8. Vonk MC, et al. Long-term follow-up results after autologous haematopoietic stem cell transplantation for severe systemic sclerosis. Ann Rheum Dis. 2008;67(1):98-104.

9. Farge D, et al. Autologous bone marrow transplantation in the treatment of refractory systemic sclerosis: early results from a French multicentre phase I-II study. Br J Haematol. 2002;119(3):726-739.

10. Prescott RJ, Freemont AJ, Jones CJ, Hoyland J, Fielding P. Sequential dermal microvascular and perivascular changes in the development of scleroderma. J Pathol. 1992;166(3):255-263.

11. Scaletti C, et al. Th2-oriented profile of male offspring T cells present in women with systemic sclerosis and reactive with maternal major histocompatibility complex antigens. Arthritis Rheum. 2002;46(2):445-450.

12. Salmon-Ehr V, et al. Expression of interleukin-4 in scleroderma skin specimens and scleroderma fibroblast cultures. Potential role in fibrosis. Arch Dermatol.1996;132(7):802-806.

13. Mavalia C, et al. Type 2 helper T-cell predominance and high CD30 expression in systemic sclerosis. Am J Pathol. 1997;151(6):1751-1758.

14. D'Elios MM, et al. In vivo CD30 expression in human diseases with predominant activation of Th2-like T cells. JLeukoc Biol. 1997;61(5):539-544.

15. Radstake TR, et al. The pronounced Th17 profile in systemic sclerosis (SSc) together with intracellular expression of TGFbeta and IFNgamma distinguishes SSc phenotypes. PLoS One. 2009;4(6):e5903.

16. Truchetet ME, Brembilla NC, Montanari E, Allanore Y, Chizzolini C. Increased frequency of circulating Th22 in addition to Th17 and Th2 lymphocytes in systemic sclerosis: association with interstitial lung disease. Arthritis Res Ther. 2011;13(5):R166.

17. Brembilla NC, Montanari E, Truchetet ME, Raschi E, Meroni P, Chizzolini C. Th17 cells favor inflammatory responses while inhibiting type I collagen deposition by dermal fibroblasts: differential effects in healthy and systemic sclerosis fibroblasts. Arthritis Res Ther. 2013;15(5):R151.

18. MacDonald KG, Dawson NA, Huang Q, Dunne JV, Levings MK, Broady R. Regulatory T cells produce profibrotic cytokines in the skin of patients with systemic sclerosis. JAllergy Clin Immunol. 2015;135(4):946-955.e9.

19. Taylor DK, et al. T follicular helper-like cells contribute to skin fibrosis. Sci Transl Med. 2018;10(431): eaaf5307.

20. Ayano M, et al. Increased CD226 expression on $\mathrm{CD}^{+} \mathrm{T}$ cells is associated with upregulated cytokine production and endothelial cell injury in patients with systemic sclerosis. J Immunol. 2015;195(3):892-900.

21. Fuschiotti P, Medsger TA, Morel PA. Effector $\mathrm{CD}^{+} \mathrm{T}$ cells in systemic sclerosis patients produce abnormally high levels of interleukin-13 associated with increased skin fibrosis. Arthritis Rheum. 2009;60(4):1119-1128.

22. Elhai M, et al. Outcomes of patients with systemic sclerosis treated with rituximab in contemporary practice: a prospective cohort study. Ann Rheum Dis. 2019;78(7):979-987.

23. Sircar G, Goswami RP, Sircar D, Ghosh A, Ghosh P. Intravenous cyclophosphamide vs rituximab for the treatment of early diffuse scleroderma lung disease: open label, randomized, controlled trial Rheumatology (Oxford). 2018;57(12):2106-2113.

24 . Taher TE, et al. Association of defective regulation of autoreactive interleukin-6-producing transitional B lymphocytes with disease in patients with systemic sclerosis. Arthritis Rheumatol. 2018;70(3):450-461.

25. Kamisawa T, Zen Y, Pillai S, Stone JH. IgG4-related disease. Lancet. 2015;385(9976):1460-1471.

26. Mattoo H, et al. Clonal expansion of CD4(+) cytotoxic $\mathrm{T}$ lymphocytes in patients with IgG4-related disease. J Allergy Clin Immunol. 2016;138(3):825-838.

27. Maehara T, et al. Lesional CD $4^{+} \mathrm{IFN}-\gamma^{+}$cytotoxic T lymphocytes in IgG4-related dacryoadenitis and sialoadenitis. Ann Rheum Dis. 2017;76(2):377-385.

28. Della-Torre E, et al. A CD $8 \alpha^{-}$subset of $\mathrm{CD}^{+}{ }^{+} \mathrm{SLAMF}^{+}$cytotoxic T cells is expanded in patients with IgG4-related disease and decreases following glucocorticoid treatment. Arthritis Rheumatol. 2018;70(7):1133-1143.

29. Soghoian DZ, Streeck H. Cytolytic CD4(+) T cells in viral immunity. Expert Rev Vaccines. 2010;9(12):1453-1463.

30. Chiu YL, et al. Cytotoxic polyfunctionality maturation of cytomegalovirus-pp65-specific $\mathrm{CD}^{+}$and $\mathrm{CD} 8^{+} \mathrm{T}$-cell responses in older adults positively correlates with response size. Sci Rep. 2016;6:19227.

31. Juno JA, van Bockel D, Kent SJ, Kelleher AD, Zaunders JJ, Munier CM. Cytotoxic CD4 T cellsfriend or foe during viral infection? Front Immunol. 2017;8:19.

32. Gilani SR, et al. CD28 down-regulation on circulating CD4 T-cells is associated with poor prognoses of patients with idiopathic pulmonary fibrosis. PLoS One. 2010;5(1):e8959.

33. Fonseka CY, et al. Mixed-effects association of single cells identifies an expanded effector CD $4^{+}$ T cell subset in rheumatoid arthritis. Sci Transl Med. 2018;10(463):eaaq0305.

34. Patil VS, et al. Precursors of human CD $4^{+}$cytotoxic T lymphocytes identified by singlecell transcriptome analysis. Sci Immunol. 2018;3(19):eaan8664. 
35. Tian Y, et al. Unique phenotypes and clonal expansions of human CD4 effector memory T cells re-expressing CD45RA. Nat Commun. 2017;8(1):1473.

36. Forestier A, et al. Altered B lymphocyte homeostasis and functions in systemic sclerosis. Autoimmun Rev. 2018;17(3):244-255.

37. Zhang J, et al. Identification of immunodominant Th2-cell epitopes in Chinese patients with bullous pemphigoid. J Invest Dermatol. 2018;138(9):1917-1924.

38. Kurane I, Meager A, Ennis FA. Dengue virusspecific human $\mathrm{T}$ cell clones. Serotype crossreactive proliferation, interferon gamma production, and cytotoxic activity. J Exp Med.1989;170(3):763-775.

39. Sharpe AH. Mechanisms of costimulation. Immunol Rev. 2009;229(1):5-11.

40. Alshekaili J, et al. STAT3 regulates cytotoxicity of human $\mathrm{CD} 7^{+} \mathrm{CD} 44^{+} \mathrm{T}$ cells in blood and lymphoid follicles. Sci Rep. 2018;8(1):3529.

41. Fernandez S, French MA, Price P. Immunosenescent $\mathrm{CD} 57^{+} \mathrm{CD} 4^{+} \mathrm{T}$-cells accumulate and contribute to interferon- $\gamma$ responses in HIV patients responding stably to ART. Dis Markers. 2011;31(6):337-342.

42. Weiskopf D, et al. Dengue virus infection elicits highly polarized CX3CR1 $1^{+}$cytotoxic $\mathrm{CD} 4^{+} \mathrm{T}$ cells associated with protective immunity. Proc Natl Acad Sci U S A. 2015;112(31):E4256-E4263.

43. Wu M, Assassi S. The role of type 1 interferon in systemic sclerosis. Front Immunol. 2013;4:266.

44. Brkic Z, et al. The interferon type I signature is present in systemic sclerosis before overt fibrosis and might contribute to its pathogenesis through high BAFF gene expression and high collagen synthesis. Ann Rheum Dis. 2016;75(8):1567-1573.

45. Dantas AT, et al. Interferons and systemic sclerosis: correlation between interferon gamma and interferon-lambda 1 (IL-29). Autoimmunity. 2015;48(7):429-433.

46. Carruthers MN, et al. Rituximab for IgG4related disease: a prospective, open-label trial. Ann Rheum Dis. 2015;74(6):1171-1177.

47. Sgonc R, Gruschwitz MS, Dietrich H, Recheis H, Gershwin ME, Wick G. Endothelial cell apoptosis is a primary pathogenetic event underlying skin lesions in avian and human scleroderma. J Clin Invest. 1996;98(3):785-792.

48. Del Papa N, et al. Antiendothelial cell antibodies induce apoptosis of bone marrow endothelial progenitors in systemic sclerosis. J Rheumatol. 2010;37(10):2053-2063.

49. Ahmed SS, Tan FK, Arnett FC, Jin L, Geng YJ. Induction of apoptosis and fibrillin 1 expression in human dermal endothelial cells by scleroderma sera containing anti-endothelial cell antibodies. Arthritis Rheum. 2006;54(7):2250-2262.

50. Yamamoto T, Nishioka K. Increased expression of p53 and p21 (Waf1/Cip1) in the lesional skin of bleomycin-induced scleroderma. Arch Dermatol Res. 2005;296(11):509-513.

51. Breuckmann F, Stuecker M, Altmeyer P, Kreuter A. Modulation of endothelial dysfunction and apoptosis: UVA1-mediated skin improvement in systemic sclerosis. Arch Dermatol Res. 2004;296(5):235-239.

52. Worda M, et al. In vivo analysis of the apoptosis-inducing effect of anti-endothelial cell antibodies in systemic sclerosis by the chorionallantoic membrane assay. Arthritis Rheum.
2003;48(9):2605-2614.

53. McMahan ZH et al. Enrichment of scleroderma vascular disease-associated autoantigens in endothelial lineage cells. Arthritis Rheumatol. 2016;68(10):2540-2549.

54. Bosurgi L, et al. Macrophage function in tissue repair and remodeling requires IL-4 or IL-13 with apoptotic cells. Science. 2017;356(6342):1072-1076.

55. Lederer DJ, Martinez FJ. Idiopathic pulmonary fibrosis. N Engl JMed. 2018;378(19):1811-1823.

56. Kissin EY, Merkel PA, Lafyatis R. Myofibroblasts and hyalinized collagen as markers of skin disease in systemic sclerosis. Arthritis Rheum. 2006;54(11):3655-3660.

57. Ecker RC, Steiner GE. Microscopy-based multicolor tissue cytometry at the single-cell level. Cytometry A. 2004;59(2):182-190.

58. Bagnoli JW, et al. Sensitive and powerful singlecell RNA sequencing using mcSCRB-seq. Nat Commun. 2018;9(1):2937.

59. Turchaninova MA, et al. High-quality full-length immunoglobulin profiling with unique molecular barcoding. Nat Protoc. 2016;11(9):1599-1616.

60. Mamedov IZ, et al. Preparing unbiased T-cell receptor and antibody cDNA libraries for the deep next generation sequencing profiling. Front Immunol. 2013;4:456.

61. Parekh S, Ziegenhain C, Vieth B, Enard W, Hellmann I. zUMIs - A fast and flexible pipeline to process RNA sequencing data with UMIs. Gigascience. 2018;7(6):giy059.

62. Newman AM, et al. Robust enumeration of cell subsets from tissue expression profiles. Nat Methods. 2015;12(5):453-457. 